DRAFT VERSION MARCH 9, 2016

Preprint typeset using LATEX style emulateapj v. 5/2/11

\title{
UPPER LIMITS FROM FIVE YEARS OF BLAZAR OBSERVATIONS WITH THE VERITAS CHERENKOV TELESCOPES
}

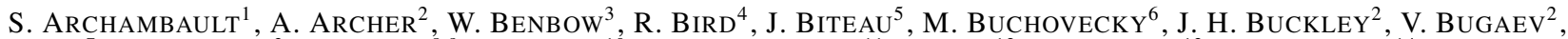

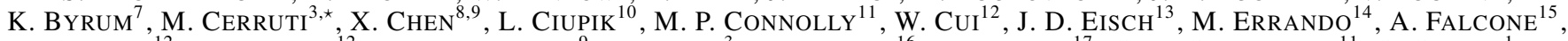

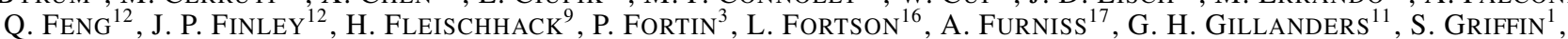
J. Grube ${ }^{10}$, G. GyuK ${ }^{10}$, M. HÜTTEN ${ }^{9}$, N. HÅKANSSON ${ }^{8}$, D. HANNA ${ }^{1}$, J. HOLder ${ }^{18}$, T. B. HuMENSKY ${ }^{19}$, C. A. JOHNSON ${ }^{5}$,

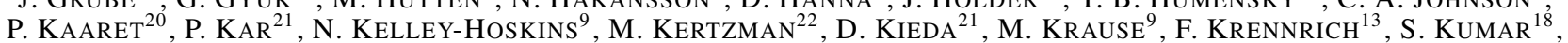

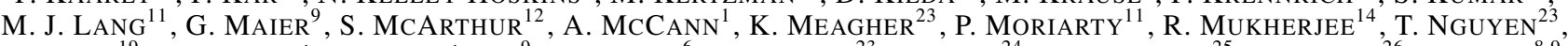
D. Nieto ${ }^{19}$, A. O'FAoláin De Bhróithe ${ }^{9}$, R. A. ONG ${ }^{6}$, A. N. OTte ${ }^{23}$, N. PARK ${ }^{24}$, J. S. PERKINS ${ }^{25}$, A. PICHEL ${ }^{26}$, M. POHL ${ }^{8,9}$,

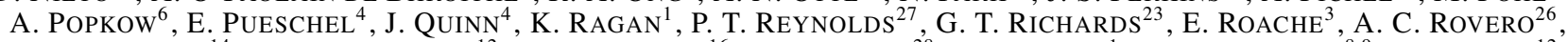
M. Santander ${ }^{14}$, G. H. Sembroski ${ }^{12}$, K. ShahinYAn ${ }^{16}$, A. W. Smith ${ }^{28}$, D. STASZAK ${ }^{1}$, I. TelezhinskY ${ }^{8,9}$, J. V. TUCCI ${ }^{12}$,

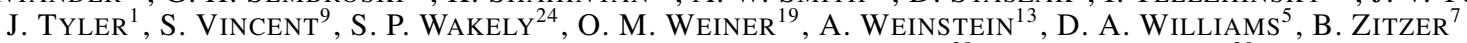
(THE VERITAS COLLABORATION), AND M. FUMAGAlli ${ }^{29}$, J. X. PROCHASKA ${ }^{30}$

Draft version March 9, 2016
\end{abstract}

\begin{abstract}
Between the beginning of its full-scale scientific operations in 2007 and 2012, the VERITAS Cherenkov telescope array observed more than 130 blazars; of these, 26 were detected as very-high-energy (VHE; E $>100$ $\mathrm{GeV}) \gamma$-ray sources. In this work, we present the analysis results of a sample of 114 undetected objects. The observations constitute a total live-time of $\sim 570$ hours. The sample includes several unidentified Fermi-Large Area Telescope (LAT) sources (located at high Galactic latitude) as well as all the sources from the second Fermi-LAT catalog which are contained within the field of view of the VERITAS observations. We have also performed optical spectroscopy measurements in order to estimate the redshift of some of these blazars that do not have a spectroscopic distance estimate. We present new optical spectra from the Kast instrument on the Shane telescope at the Lick observatory for 18 blazars included in this work, which allowed for the successful measurement or constraint on the redshift of four of them. For each of the blazars included in our sample we provide the flux upper limit in the VERITAS energy band. We also study the properties of the significance distributions and we present the result of a stacked analysis of the data-set, which shows a $4 \sigma$ excess.
\end{abstract}

Subject headings: BL Lacertae objects: general - galaxies: active - gamma rays: galaxies - radiation mechanisms: non-thermal

\footnotetext{
${ }^{1}$ Physics Department, McGill University, Montreal, QC H3A 2T8, Canada

${ }^{2}$ Department of Physics, Washington University, St. Louis, MO 63130, USA

${ }^{3}$ Fred Lawrence Whipple Observatory, Harvard-Smithsonian Center for Astrophysics, Amado, AZ 85645, USA

wystan.benbow@cfa.harvard.edu

${ }^{4}$ School of Physics, University College Dublin, Belfield, Dublin 4, Ireland

${ }^{5}$ Santa Cruz Institute for Particle Physics and Department of Physics, University of California, Santa Cruz, CA 95064, USA caajohns@ucsc.edu

${ }^{6}$ Department of Physics and Astronomy, University of California, Los Angeles, CA 90095, USA

${ }^{7}$ Argonne National Laboratory, 9700 S. Cass Avenue, Argonne, IL 60439, USA

${ }^{8}$ Institute of Physics and Astronomy, University of Potsdam, 14476 Potsdam-Golm, Germany

${ }^{9}$ DESY, Platanenallee 6, 15738 Zeuthen, Germany

${ }^{10}$ Astronomy Department, Adler Planetarium and Astronomy Museum, Chicago, IL 60605, USA

${ }^{11}$ School of Physics, National University of Ireland Galway, University Road, Galway, Ireland

${ }^{12}$ Department of Physics and Astronomy, Purdue University, West Lafayette, IN 47907, USA

${ }^{13}$ Department of Physics and Astronomy, Iowa State University, Ames, IA 50011, USA

${ }^{14}$ Department of Physics and Astronomy, Barnard College, Columbia University, NY 10027, USA

15 Department of Astronomy and Astrophysics, 525 Davey Lab, Pennsylvania State University, University Park, PA 16802, USA

${ }^{16}$ School of Physics and Astronomy, University of Minnesota, Min-
}

neapolis, MN 55455, USA

${ }^{17}$ Department of Physics, California State University - East Bay, Hayward, CA 94542, USA

${ }^{18}$ Department of Physics and Astronomy and the Bartol Research Institute, University of Delaware, Newark, DE 19716, USA

${ }^{19}$ Physics Department, Columbia University, New York, NY 10027, USA

${ }^{20}$ Department of Physics and Astronomy, University of Iowa, Van Allen Hall, Iowa City, IA 52242, USA

${ }^{21}$ Department of Physics and Astronomy, University of Utah, Salt Lake City, UT 84112, USA

${ }^{22}$ Department of Physics and Astronomy, DePauw University, Greencastle, IN 46135-0037, USA

${ }^{23}$ School of Physics and Center for Relativistic Astrophysics, Georgia Institute of Technology, 837 State Street NW, Atlanta, GA 30332-0430

${ }^{24}$ Enrico Fermi Institute, University of Chicago, Chicago, IL 60637, USA

${ }^{25}$ N.A.S.A./Goddard Space-Flight Center, Code 661, Greenbelt, MD 20771, USA

${ }^{26}$ Instituto de Astronomia y Fisica del Espacio, Casilla de Correo 67 Sucursal 28, (C1428ZAA) Ciudad Autónoma de Buenos Aires, Argentina

${ }^{27}$ Department of Physical Sciences, Cork Institute of Technology, Bishopstown, Cork, Ireland

${ }^{28}$ University of Maryland, College Park / NASA GSFC, College Park, MD 20742, USA

${ }^{29}$ Institute for Computational Cosmology and Centre for Extragalactic Astronomy, Department of Physics, Durham University, South Road, Durham, DH1 3LE, UK

${ }^{30}$ Department of Astronomy and Astrophysics, University of California, Santa Cruz, CA 95064, USA

* Now at Sorbonne Universités, UPMC Université Paris 06, Université Paris Diderot, Sorbonne Paris Cité, CNRS, Laboratoire de Physique Nu- 


\section{INTRODUCTION}

The current generation of imaging atmospheric Cherenkov telescopes (IACTs), sensitive to very-high-energy (VHE; $\mathrm{E}>100 \mathrm{GeV}) \gamma$-ray photons, has significantly increased our knowledge of blazars. They represent by far the class of objects which dominates the VHE extragalactic sky. Among the 66 extragalactic VHE sources currently detected 11 about $90 \%$ of them are blazars.

In the framework of the unified model of active galactic nuclei (AGN), blazars are radio-loud AGN, characterized by a pair of relativistic jets of plasma emitted along the polar axis of the super-massive black-hole powering the system, aligned along the line of sight of the observer (see Urry \& Padovani 1995). The observational properties of blazars include a spectral energy distribution (SED) characterized by a non-thermal continuum from radio to $\gamma$-rays, extreme temporal variability and strong polarization. These properties can be explained by considering that the emission from the jet, enhanced by relativistic effects, dominates the SED (Angel \& Stockman 1980). Spectroscopic measurements in optical and UV reveal that two distinct sub-classes of blazars exist: BL Lac objects, characterized by a featureless optical/UV spectrum, and flat-spectrum radio-quasars (FSRQs), which show instead broad emission lines. The traditional division between the two classes of objects is an equivalent width of the emission lines equal to $5 \AA$ (see Stickel et al. 1991). The two sub-classes are also characterized by different luminosity and redshift distributions. The FSRQs are on average brighter and located at higher redshifts (see e.g. Padovani 1992, Massaro et al. 2009). In the unified AGN model, this dichotomy is associated with a similar dichotomy seen in radio-galaxies. FSRQs are considered the blazar version of FR II radio-galaxies (Fanaroff \& Riley 1974), while BL Lac objects correspond to FR I (Urry \& Padovani 1995).

Two broad non-thermal components characterize blazar SEDs. The first, peaking between millimeter and X-rays, is attributed to synchrotron emission by a population of electrons/positrons in the blazar jet. The second, peaking in $\gamma$-rays, is associated, in leptonic models, with inverse Compton scattering between the same leptons and their own synchrotron emission (synchtron-self-Compton model, SSC, Konigl 1981), or an external photon field, such as the emission from the dusty torus, the accretion disc or the broad-line-region (see Sikora et al. 1994). Alternatively, in hadronic scenarios, the second SED component is attributed to synchrotron emission by protons, or by secondary particles produced in $\mathrm{p}-\gamma$ interactions (Mücke \& Protheroe 2001). The position of the first peak is used to further classify BL Lac objects into low and high-frequency-peaked BL Lac objects (LBL/HBL), depending on whether the peak frequency is located in infrared/optical or UV/X-rays, respectively. The transition between LBL and HBL is smooth, and a population of intermediate-frequency-peaked BL Lac objects exists as well (IBL, with $10^{14} \mathrm{~Hz}<\nu_{\text {syn }}<10^{15} \mathrm{~Hz}$, see Laurent-Muehleisen et al. 1998). While BL Lac objects

cléaire et de Hautes Energies (LPNHE), 4 place Jussieu, F-75252, Paris Cedex 5, France

matteo.cerruti@lpnhe.in2p3.fr

${ }^{1}$ For a recent review, see e.g. Şentürk et al.(2013); for an updated list of known VHE sources see http://tevcat.uchicago.edu present a variety of synchrotron peak frequencies, FSRQs are almost all characterized by a low-frequency peak. This classification can be seen as a more recent version of the older classification (see e.g. Padovani \& Giommi 1995b) of blazars into radio-selected objects (RBLs, which are more likely FSRQs and LBLs) and X-ray-selected objects (XBLs, which are more likely HBLs)

The measurement of blazar spectral properties at VHE is important not only to characterize the blazar emission itself, but also to indirectly study the extragalactic background-light (EBL) in the infrared and visible bands due to the absorption that it causes on VHE photons via $e^{ \pm}$pair-production, (see Salamon \& Stecker 1998). VHE observations can also be used to put limits on the strength of the intergalactic magnetic field according to the non-detection of the emission from the cascade triggered by the interaction of the pairs with the cosmic-microwave-background (see e.g. Taylor et al.2011).

Even though the detection and the measurement of the VHE spectrum of a blazar is of paramount importance for the comprehension of the physics of relativistic jets in AGN and for cosmological studies, a non-detection in the VHE regime can also be extremely useful. It can constrain the source emission model, especially when the flux upper-limit is significantly lower than the extrapolation of the Fermi-LAT (Atwood et al. 2009) measurement in high-energy $\gamma$-rays (HE; $100 \mathrm{MeV}<\mathrm{E}<100 \mathrm{GeV}$ ) up to $\mathrm{VHE}$, implying the presence of a spectral cut-off. It can also constrain the variability properties of the source at VHE if the blazar has been previously detected or is detected at a later time during a higher flux state. Additionally, for the upcoming Cherenkov Telescope Array (CTA, Actis et al. 2011), it will be useful to have the information from all past observations performed by the current generation of IACTs, in order to make predictions for expected outcomes (see e.g. Sol et al. 2013).

All three major IACTs (H.E.S.S., MAGIC and VERITAS) have published upper-limits on blazar VHE emission, including more than 70 sources in their lists (Aharonian et al. 2005, 2008b; Aleksić et al. 2011a; Aliu et al. 2012, Abramowski et al.2014). Past IACTs, such as CAT, HEGRA and Whipple, have also presented the results from undetected blazars (Piron 2000; Aharonian et al. 2004; Falcone et al. 2004; Horan et al. 2004), though their limits have in general been superseded by the current instruments. At higher energies, upper limits on blazars have also been estimated using the air-shower technique with Milagro (Williams 2005).

The study of blazars is complicated by the uncertainty in their redshifts. In fact, the almost featureless spectra of BL Lac objects imply that a redshift estimate can be obtained only via absorption features from the host galaxy or clouds in the intergalactic medium, or via molecular emission lines (see Fumagalli et al. 2012), or via a less precise photometric estimate (comparing the luminosity of the host galaxy to samples of giant elliptical galaxies). If the blazar belongs to a group of galaxies, its redshift can also be estimated by studying the non-active companions (see Muriel et al.2015).

2 The classification of BL Lac objects as LBL/IBL/HBL is sometimes replaced by LSP/ISP/HSP (low/intermediate/high-synchrotron-peaked blazars, see Abdo et al. 2010b), making explicit reference to the synchrotron origin of the first component of the SED. For BL Lac objects, the two triplets of acronyms can be considered as synonyms. 
For VHE studies, the knowledge of the redshift of the blazar is very important, because the absorption by the EBL increases with the distance of the object. Currently, the farthest detected VHE blazar is the gravitationally-lensed quasar S3 0218+357 (Mirzoyan 2014b) at $z=0.944$, closely followed by the FSRQ PKS 1441+25 (Ahnen et al. 2015; Abeysekara et al. 2015) at $z=0.939$, while the farthest, persistent (i.e. detected not only during episodic flaring activity) VHE blazar is PKS 1424+240 $(z>0.61$, see Acciari et al. 2010, Furniss et al. 2013, Archambault et al. 2014). To improve the constraints on the distance of some VHE candidates, new redshift estimates obtained with the Kast spectrograph at Lick Observatory (see Section 2 and Appendix A are presented together with the VHE upper limits from VERITAS.

In this paper we present the results of the analysis of most of the non-detected blazars observed by VERITAS from 2007 (the beginning of full-scale scientific operations) to August 2012 (before the upgrade of the VERITAS array, see Kieda 2013). VERITAS upper limits on six VHE candidates were presented by Aliu et al. (2012). The sample also includes several unidentified Fermi-LAT objects (located at high Galactic latitude, and most probably associated with unidentified AGN, see Ackermann et al.2012a; Mirabal et al. 2012, Doert \& Errando 2014).

The paper is organized as follows: in Section 2 we provide the details of the properties of the sample and the source selection; the details of the VERITAS data analysis and results are provided in Section 3, a stacked analysis of the data is presented in Section 4, studying the full data-set as well as sub-data-sets defined by redshifts and blazar classes; the conclusions are in Section 5 .

\section{THE SAMPLE AND NEW REDSHIFT ESTIMATES}

Blazars targeted by VERITAS as VHE source candidates were selected according to a variety of criteria. Early source selections were based on blazar X-ray or radio catalogs, while more recent candidates have also been selected on the basis of their Fermi-LAT spectral characteristics (Abdo et al. 2009a, 2010a, Nolan et al. 2012) or on their association with clusters of $\mathrm{HE} \gamma$-rays (see Archambault et al. 2013). The target list includes:

- all the nearby $(z<0.3)$ HBL/IBL recommended as potential VHE emitters by Stecker et al. (1996); Perlman (2000) and Costamante \& Ghisellini (2002)

- the X-ray brightest nearby $(z<0.3) \mathrm{HBL}$ in the Sedentary (Giommi et al. 2005) and ROXA (Turriziani et al. 2007) surveys

- four distant $(z>0.3)$ BL Lac objects recommended by Costamante \& Ghisellini (2002) and Costamante (2007)

- all nearby $(z<0.3)$ blazars detected by EGRET (Mukherjee 2001)

- several FSRQs recommended as potential VHE emitters by Perlman (2000) and Padovani et al. (2002)

- two high-frequency-peaked FSRQs (B2 0321+33 and Mrk 1218, see Perlman 2000; Falcone et al. 2004), which have also been classified as Seyfert-1 galaxies (see Osterbrock \& Dahari 1983, Abdo et al. 2009b)

- the brightest Fermi-LAT sources after extrapolation into the VERITAS energy band (Abdo et al. 2009a 2010a, Nolan et al. 2012)

- sources associated with clusters of HE $\gamma$-rays, but not included in Fermi-LAT catalogs, similar to VER J0521+211 (Archambault et al. 2013).

In addition, several targets have been observed by VERITAS only as targets of opportunity (ToO), following flare alerts by multi-wavelength partners (see Errando 2011).

The sources included in our sample are listed in Table 1 (for sources without VHE detection, 82 targets) and Table 2 (for known VHE emitters, detected by other instruments, or by VERITAS after 2012 and during flaring activity, 11 targets). Sources are listed in order of increasing Right Ascension (R.A.). For every target, we indicate the name, the coordinates (R.A. and Dec, in J2000), the catalog redshift $z$, the blazar class, the VERITAS dead-time corrected exposure (see Section 3), the average zenith angle of VERITAS observations, the dates of VERITAS observations (in MJD) and on which basis the source was selected as a VHE candidate. Names and coordinates are taken from the SIMBAD database ${ }^{3}$ The references for the redshift estimates (and their uncertainties) and the blazar class are provided in the table notes. For every source, the archival SED from the ASDC $S E D$ Builder too ${ }^{4}$ has been visually inspected. It is used to classify all BL Lac objects marked as HBLs. The total number of hours of VERITAS data analyzed is about 570, which represents about $60 \%$ of a single VERITAS yearly observing season, i.e. about one tenth of all good quality VERITAS data taken from 2007 to 2012.

The field-of-view (FoV) of the VERITAS telescope array is about $3.5^{\circ}$ and for every observation there is a chance that, in addition to the targeted blazar, other $\gamma$-ray sources are contained within the FoV. For every target included in our sample we checked if other known $\gamma$-ray sources (included in the 2-year Fermi-LAT catalog, 2FGL, see Nolan et al. 2012) were present in the FoV. Twenty-one 2FGL sources were indirectly observed by VERITAS through proximity to the blazar of interest, and are listed in Table 3 . We indicate as well the counterpart name (from the 2FGL catalog), the coordinates (R.A. and Dec) of the counterpart, the redshift and the blazar sub-class (if known). The majority of these additional 2FGL sources are AGN without classification.

\subsection{New redshift measurements}

In order to measure the distance of some $\gamma$-ray blazars, we observed 18 of the VERITAS targets using the dual-arm Kast spectrograph at the Cassegrain focus of the Shane telescope at Lick Observatory. For all the observations presented here, the instrument was configured with the 600/4310 grism on the blue arm, and the 600/7500 grating on the red arm, the D55 dichroic, and a $2^{\prime \prime}$ slit. The dichroic crossover

3 http://simbad.u-strasbg.fr/simbad/

4 http://tools.asdc.asi.it/SED/ 
creates an instrumental gap located at $\sim 5500 \AA$ and affects approximately $200 \AA$ of the spectrum. In the spectroscopic figures A1 - A6 (Appendix A), this gap is shown on each spectrum. While the absolute fluxes are shown in the plots, the flux calibration is the least certain aspect of the spectra. In several spectra there is a gap in flux across the dichroic; this is purely the result of calibrations and is not intrinsic to the AGN. The wavelength coverage is from $\sim 3450 \AA$ to $\sim 8000 \AA$, but tellurics and fringing mask features above $6850 \AA$. We do not show this contaminated portion in the spectra. The targets, observation dates and exposures are summarized in Table A1. The corresponding standard star and the signal-to-noise ratio $(S / N)$ are also included in this table. The data were reduced following standard techniques with the Low-Redux pipelin $6^{5}$. Each spectrum was inspected visually for absorption or emission features. Features are noted in the spectral plots, Figures A1 - A6 and in Table A1 For 14 of the sources observed, there are no spectral features that allow redshift measurements or redshift limits. By definition, BL Lac objects have no or weak spectral lines, so this high rate of non-detections is somewhat expected. Below are the sources for which features were found that allow for redshift determinations (see Figure A1):

\section{- RGB J0250+172}

Observations of the source were obtained on August 15,2010 (UT) and resulted in the detection of galactic features at $z=0.243$. We detected $\mathrm{Ca}$ II $(\mathrm{H}$, equivalent width $(\mathrm{EW})=870 \pm 160 \mathrm{m \AA} ; \mathrm{K}, E W=1340 \pm$ $190 \mathrm{~m} \AA), \mathrm{G}$ band $(E W=650 \pm 180 \mathrm{~m} \AA)$ and $\mathrm{Mg} \mathrm{I}$ $(E W=530 \pm 120 \mathrm{~m} \AA)$ absorption.

In the literature, Bauer et al. (2000) quotes a redshift of $\mathrm{z}=1.10$ for RGB J0250+172; however, there is no information on spectroscopic lines provided within the reference. Nilsson et al. (2003) presents optical images of BL Lac objects, including RGB J0250+172. They find that the object is clearly resolved and state that $z=1.10$ is too high because it results in a host galaxy that is exceedingly bright $\left(M_{R}<-29.0\right)$. Based on fits to the observed light profile, a redshift estimate of $z=0.25$ is provided, which is similar to the value measured within this work.

\section{- 1ES 1118+424}

Observations of the source were obtained on February 14, 2013 (UT) and resulted in the detection of galactic features at $z=0.230$. We detected $\mathrm{Ca}$ II $(\mathrm{H}$, $E W=11500 \pm 1500 \mathrm{~m} \AA ; \mathrm{K}, E W=13000 \pm 1700 \mathrm{~m} \AA$ ), $\mathrm{G}$ band $(E W=3790 \pm 862 \mathrm{~m} \AA), \mathrm{Ca} \mathrm{I}(E W=1208 \pm$ $199 \mathrm{m \AA})$ and $\mathrm{Mg} \mathrm{I}(E W=2896 \pm 254 \mathrm{~m} \AA)$ absorption lines.

In the literature, the redshift for $1 \mathrm{ES} 1118+424$ is quoted as $z=0.124$ from a private communication (see Falomo \& Kotilainen 1999). However, Falomo \& Kotilainen (1999) derive a lower limit of $z>0.5$ based on images taken using the Nordic Optical Telescope, where the source is unresolved. They simulate an

\footnotetext{
5 http://www.ucolick.org/ xavier/LowRedux/
}

elliptical host galaxy with $M_{R}=-23.8$ and an effective radius of $10 \mathrm{kpc}$ to determine the lowest redshift at which it would not be resolved. These galactic parameters are what they find from other BL Lac objects in their study, and they note that assuming a less luminous and smaller host galaxy would result in a lower redshift estimate.

\section{- RBS 1366 (=1E 1415+25.9)}

Observations of the source obtained on May 30, 2014 (UT) resulted in the detection of galactic features at $z=$ 0.237 . We detected Ca II (H, $E W=1300 \pm 130 \mathrm{~mA} ; \mathrm{K}$, $E W=1570 \pm 140 \mathrm{~m} \AA), \mathrm{G}$ band $(E W=210 \pm 160 \mathrm{~m} \AA)$, $\mathrm{Ca} I(E W=710 \pm 64 \mathrm{m \AA})$ and $\mathrm{Mg} \mathrm{I}(E W=1900 \pm$ $100 \mathrm{~m} \AA$ ) absorption.

Halpern et al. (1986) also measure a redshift of $z=0.237$ based on Ca II, G band, Fe I, Mg I and $\mathrm{Na}$ absorption. This source displays the significant variability associated with BL Lac objects. The spectrum taken in 2013 has a lower overall flux than the spectrum taken in 2014 (see Table A1), indicating that the source might have been in different flux states.

\section{- 1ES 2321+419}

Observations of the source obtained on October 28, 2014 (UT) resulted in the detection of absorption features at $z=0.267$. We detected $\mathrm{Ca}$ II $(\mathrm{H}, E W=260 \pm$ $47 \mathrm{~m} \AA ; \mathrm{K}, E W=180 \pm 52 \mathrm{m \AA})$ and $\mathrm{Mg}$ II $(2796 \AA$, $E W=740 \pm 83 \mathrm{~m} \AA ; 2803 \AA$, $E W=510 \pm 67 \mathrm{m \AA}) \mathrm{ab}-$ sorption. Because the Ca II absorption is narrow, and there are no other galactic features, only a lower limit can be set on the redshift of the source. Additionally, there is potentially $\mathrm{Mg}$ II absorption at a higher redshift, $z=0.346$.

In the literature, Falomo \& Kotilainen (1999) derive a lower limit for this source of $z>0.45$ using the same technique as for 1ES $1118+424$. While our value is not inconsistent, it is considerably lower than that placed based on assumptions about the host galaxy.

\section{VERITAS OBSERVATIONS AND DATA ANALYSIS}

The VERITAS (Very Energetic Radiation Imaging Telescope Array System) telescope array is composed of four IACTs of 12-m diameter each, located at the Fred Lawrence Whipple Observatory, on the slopes of Mount Hopkins, in southern Arizona ( $\left.31^{\circ} 40^{\prime} \mathrm{N}, 110^{\circ} 57^{\prime} \mathrm{W}\right)$. Each telescope has a segmented mirror which focuses light onto a camera composed of 499 photomultipliers located at the focal plane. The instrument FoV is $3.5^{\circ}$. For further details on the VERITAS instrument see Holder et al. (2006); Holder (2011).

The telescopes measure the faint Cherenkov light induced by the electromagnetic showers triggered by the interaction of the $\gamma$-ray photons with the Earth atmosphere. Similar cascades triggered by cosmic rays are also detected by VERITAS, and can be rejected by applying specific cuts on the shape of the Cherenkov image (Hillas 1985). 
Table 1

List of sources observed by VERITAS

\begin{tabular}{|c|c|c|c|c|c|c|c|c|}
\hline Source name & R.A. & Dec & $z^{a}$ & Type $^{b}$ & Exposure & $\hat{\theta}_{\text {zenith }}$ & MJDs & Selection $^{c}$ \\
\hline & [hr min sec] & [deg min sec] & & & [hr] & {$[\mathrm{deg}]$} & {$[-50000]$} & \\
\hline RBS 0042 & 001827.8 & +294732 & $0.100:^{1}$ & $\mathrm{HBL}$ & 7.1 & 15 & $\begin{array}{c}4731 / 32 / 33 / 40 \\
4741 / 42 / 46 / 73 \\
5089 / 90 / 91 \\
5131 / 43\end{array}$ & SHBL \\
\hline RBS 0082 & 003514.7 & +151504 & $1.28:^{2}$ & $\mathrm{HBL}$ & 6.3 & 19 & $\begin{array}{c}5100 / 01 / 02 \\
5119 / 20 / 29 / 30\end{array}$ & SHBL \\
\hline 1ES $0037+405$ & 004013.8 & +405005 & - & HBL & 36.0 & 14 & $\begin{array}{c}4767 / 68 / 71 / 72 \\
4773 / 89 / 91 \\
4800 / 02 / 22 / 29 / 46 \\
5156 / 57 / 58 / 59 \\
5457 / 70 / 71 / 72 \\
5475 / 76 / 77 / 78 / 79 \\
5495 / 96 / 97 / 98 \\
5500 / 01 / 23 / 26 \\
5546 / 54 / 56\end{array}$ & $\mathrm{ToO}$ \\
\hline 1RXS J0045.3+2127 & 004519.2 & +212743 & - & HBL & 1.2 & 22 & 5512 & N06, 1FGL \\
\hline RGB J0110+418 & 011004.9 & +414951 & $0.096^{3}$ & $\mathrm{IBL}^{38}$ & 4.0 & 13 & $\begin{array}{c}4832 / 33 \\
5866 / 67 / 68 / 96\end{array}$ & $\mathrm{P} 00$ \\
\hline $1 \mathrm{ES} 0120+340$ & 012308.7 & +342051 & $0.272^{4}$ & HBL & 5.9 & 16 & $\begin{array}{c}4383 / 84 / 93 / 94 \\
4414 / 37 / 5171 \\
5470 / 73 / 99 \\
5512 / 26\end{array}$ & SHBL, CG02 \\
\hline QSO $0133+476$ & 013658.6 & +475129 & $0.859^{5}$ & $\mathrm{FSRQ}^{39}$ & 0.8 & 40 & 4508 & $\mathrm{ToO}$ \\
\hline B2 $0200+30$ & 020345.6 & +304130 & $0.761^{6}$ & - & 1.8 & 21 & $5569 / 70 / 88$ & $\mathrm{ToO}$ \\
\hline CGRaBS J0211+1051 & 021113.1 & +105135 & $0.20:^{7}$ & $\mathrm{LBL}^{40}$ & 4.0 & 31 & $\begin{array}{c}5588 / 89 / 90 / 91 \\
5595 / 99\end{array}$ & $\mathrm{ToO}$ \\
\hline RGB J0214+517 & 021417.9 & +514452 & $0.049^{8}$ & $\mathrm{IBL}^{41}$ & 5.1 & 22 & $\begin{array}{c}4773 / 89 / 90 / 91 / 94 \\
4800 / 5156 / 81 \\
5201 / 04 / 5489\end{array}$ & P00, CG02 \\
\hline RBS 0298 & 021630.9 & +231513 & $0.289^{9}$ & HBL & 3.1 & 14 & $4731 / 32 / 33 / 73$ & SHBL \\
\hline RBS 0319 & 022716.6 & +020200 & $0.457^{10}$ & HBL & 0.3 & 31 & 4412 & SHBL \\
\hline $\mathrm{AO} 0235+16$ & 023838.9 & +163659 & $0.94^{11}$ & $\mathrm{LBL}^{41}$ & 4.3 & 21 & $4737 / 38 / 39 / 42 / 45$ & 1FGL, ToO \\
\hline RGB J0250+172 & 025038.0 & +171208 & $0.243^{12}$ & $\mathrm{IBL}^{38}$ & 5.1 & 22 & $\begin{array}{c}5144 / 59 / 5472 \\
5528 / 42 / 71 / 98 \\
\end{array}$ & 1FGL \\
\hline 2FGL J0312.8+2013 & 031223.0 & +200750 & - & - & 9.7 & 14 & $\begin{array}{c}5209 \\
5830 / 33 / 34 / 40 \\
5855 / 56 / 57 / 58 \\
5860 / 61 / 62 \\
\end{array}$ & $2 \mathrm{FGL}$ \\
\hline RGB J0314+247 & 031402.7 & +244433 & $0.056^{3}$ & $\mathrm{LBL}^{42}$ & 3.1 & 28 & $\begin{array}{c}4441 \\
4761 / 62 / 63 / 64 / 73\end{array}$ & $\mathrm{P} 00$ \\
\hline RGB J0314+063 & 031423.9 & +061957 & - & HBL & 0.3 & 26 & 5868 & SHBL \\
\hline RGB J0321+236 & 032200.0 & +233611 & - & $\mathrm{IBL}^{38}$ & 9.2 & 12 & $\begin{array}{c}5501 / 02 / 03 / 04 \\
5507 / 08 / 10 / 11 \\
5512 / 13 / 14\end{array}$ & 1FGL \\
\hline B2 $0321+33$ & 032441.2 & +341046 & $0.061^{8}$ & $\begin{array}{l}\text { FSRQ/ } \\
\text { NLS1 }^{43}\end{array}$ & 9.1 & 12 & $\begin{array}{l}4409 / 12 / 16 / 37 \\
4438 / 39 / 40 / 47 \\
4448 / 49 / 50 / 64\end{array}$ & P00, F04 \\
\hline 1FGL J0333.7+2919 & 033349.2 & +291632 & - & $\mathrm{IBL}^{44}$ & 0.6 & 3 & 5571 & 1FGL \\
\hline 1RXS J044127.8+150455 & 044127.4 & +150456 & $0.109^{13}$ & $\mathrm{HBL}$ & 10.1 & 21 & $\begin{array}{c}4747 / 48 / 49 / 89 / 90 \\
4831 / 32 / 33 \\
4847 / 49 / 51 / 79 \\
4880 / 82 / 91\end{array}$ & SHBL \\
\hline 2FGL J0423.3+5612 & 042327.0 & +561224 & - & - & 1.6 & 28 & $5855 / 5926$ & 2 FGL \\
\hline 1FGL J0423.8+4148 & 042356.1 & +415003 & - & - & 1.0 & 15 & 5599 & 1FGL \\
\hline 1ES 0446+449 & 045007.3 & +450312 & $0.203:^{4}$ & $\mathrm{IBL}^{38}$ & 7.1 & 22 & $\begin{array}{c}4734 / 42 / 61 / 62 \\
4763 / 64 / 65 \\
5209 / 13 / 34 / 35 \\
5838\end{array}$ & S96 \\
\hline RGB J0505+612 & 050558.7 & +611336 & - & - & 9.2 & 32 & $\begin{array}{c}5502 / 03 / 29 / 30 \\
5535 / 36 / 40 / 41 \\
5543 / 44 / 57 / 58 / 72\end{array}$ & 1FGL \\
\hline 1FGL J0515.9+1528 & 051547.3 & +152717 & - & - & 3.9 & 18 & $\begin{array}{c}5480 / 81 / 82 \\
5558 / 59\end{array}$ & 1FGL \\
\hline 2FGL J0540.4+5822 & 054026.0 & +582254 & - & - & 1.3 & 30 & $5856 / 5926 / 27$ & 2FGL \\
\hline RGB J0643+422 & 064326.8 & +421419 & $0.080^{14}$ & HBL & 1.2 & 23 & $4439 / 4790 / 4892$ & B97 \\
\hline RGB J0656+426 & 065610.7 & +423702 & $0.061^{15}$ & $\mathrm{IBL}^{37}$ & 9.4 & 16 & $\begin{array}{c}4746 / 66 / 67 / 77 \\
4778 / 79 / 90 / 4800\end{array}$ & $\mathrm{P} 00$ \\
\hline 1ES 0735+178 & 073807.4 & +174219 & $0.424^{16}$ & $\mathrm{IBL}^{44}$ & 5.2 & 18 & $\begin{array}{c}5531 / 32 / 58 / 59 \\
5574 / 87 / 5602\end{array}$ & 1FGL \\
\hline BZB J0809+3455 & 080938.9 & +345537 & $0.082^{8}$ & HBL & 1.6 & 13 & $5928 / 29 / 30$ & 1FGL \\
\hline PKS 0829+046 & 083148.9 & +042939 & $0.174^{17}$ & $\mathrm{IBL}^{37}$ & 2.4 & 30 & $4822 / 4921 / 5181$ & M01 \\
\hline
\end{tabular}


Table 1 - Continued

\begin{tabular}{|c|c|c|c|c|c|c|c|c|}
\hline Source name & R.A. & Dec & $z^{a}$ & Type $^{b}$ & Exposure & $\hat{\theta}_{\text {zenith }}$ & MJDs & Selection $^{c}$ \\
\hline & [hr min sec] & [deg min sec] & & & [hr] & {$[\mathrm{deg}]$} & {$[-50000]$} & \\
\hline Mrk 1218 & 083810.9 & +245343 & $0.028^{18}$ & $\begin{array}{l}\text { FSRQ/ } \\
\text { Sy } 1^{45}\end{array}$ & 5.9 & 13 & $\begin{array}{l}4423 / 25 / 39 / 40 \\
4448 / 49 / 50 / 52\end{array}$ & F04 \\
\hline OJ 287 & 085448.9 & +200631 & $0.306^{19}$ & $\mathrm{LBL}^{46}$ & 10.2 & 19 & $\begin{array}{c}4438 / 39 / 40 / 48 \\
4449 / 50 / 52 / 65 / 66 \\
5233 / 35 / 37 / 66 \\
5302 \\
\end{array}$ & $\begin{array}{l}\text { CG02, M01 } \\
\text { ToO }\end{array}$ \\
\hline B2 0912+29 & 091552.4 & +293324 & $0.36:^{7}$ & HBL & 11.7 & 10 & $\begin{array}{c}5571 / 72 / 74 / 75 \\
5576 / 87 / 88 / 89 / 90 \\
5630 / 44 / 72 / 73\end{array}$ & 1FGL \\
\hline 1ES $0927+500$ & 093037.6 & +495026 & $0.188^{4}$ & HBL & 11.7 & 23 & $\begin{array}{c}4466 / 4770 / 71 \\
4800 / 01 / 02 / 03 / 06 \\
4807 / 20 / 21 / 22 \\
5247 / 75\end{array}$ & SHBL, ROXA \\
\hline RBS 0831 & 100811.4 & +470522 & $0.343^{20}$ & HBL & 1.6 & 18 & $5531 / 59 / 89$ & SHBL, ROXA \\
\hline RGB J1012+424 & 101244.3 & +422957 & $0.36:^{21}$ & $\mathrm{IBL}^{37}$ & 1.7 & 15 & $5589 / 5931 / 86$ & ROXA \\
\hline 1ES $1028+511$ & 103118.5 & +505336 & $0.36:^{10}$ & HBL & 24.1 & 23 & $\begin{array}{c}4412 / 13 / 15 / 4530 \\
4828 / 29 / 30 / 31 \\
4832 / 59 / 83 \\
4905 / 06 / 11 / 21 \\
4922 / 23 / 27 / 28 \\
5292 / 93 / 95 / 98 \\
5301 / 03 \\
5919 / 23 / 27 / 31 / 45 \\
5946 / 58 / 70 / 79 / 82 \\
6000 / 02 / 09 / 27 \\
6035 / 38\end{array}$ & $\begin{array}{l}\text { SHBL, CG02 } \\
\text { ROXA }\end{array}$ \\
\hline RGB J1037+571 & 103744.3 & +571156 & $>0.62:^{7}$ & $\mathrm{IBL}^{37}$ & 3.7 & 26 & $5241 / 42 / 43 / 46$ & 1FGL \\
\hline RGB J1053+494 & 105344.1 & +492956 & $0.140^{22}$ & HBL & 7.8 & 24 & $\begin{array}{c}4879 / 81 / 82 / 88 / 91 \\
4921 / 22 \\
5157 / 58 / 59\end{array}$ & 1FGL \\
\hline RBS 0921 & 105606.6 & +025214 & $0.236^{23}$ & HBL & 2.7 & 30 & $4821 / 22 / 51$ & SHBL \\
\hline RBS 0929 & 110021.1 & +401928 & - & HBL & 4.4 & 16 & $5333 / 5587 / 88 / 89$ & SHBL, ROXA \\
\hline 1ES 1106+244 & 110916.2 & +241120 & $0.482^{24}$ & HBL & 1.0 & 17 & $5981 / 82$ & $\mathrm{C} 07$ \\
\hline RX J1117.1+2014 & 111706.3 & +201407 & $0.139^{15}$ & HBL & 9.1 & 16 & $\begin{array}{l}4940 / 41 / 42 / 5538 \\
5540 / 41 / 42 / 43 / 44 \\
5543 / 63 / 64 / 65 / 71\end{array}$ & $\begin{array}{l}\text { SHBL, CG02 } \\
\text { 1FGL, ToO }\end{array}$ \\
\hline 1ES $1118+424$ & 112048.1 & +421212 & $0.230^{12}$ & HBL & 6.4 & 17 & $\begin{array}{c}5212 / 32 / 74 / 75 \\
5276 / 89 / 90 / 91\end{array}$ & SHBL, S96 \\
\hline S4 1150+497 & 115324.5 & +493109 & $0.334^{25}$ & FSRQ $^{38}$ & 3.8 & 24 & $\begin{array}{l}5701 / 02 / 03 / 04 / 05 \\
5706 / 07 / 08 / 09 / 10\end{array}$ & ROXA, ToO \\
\hline RGB J1231+287 & 123143.6 & +284750 & $1.03^{26}$ & HBL & 5.1 & 17 & $\begin{array}{c}5239 / 66 / 91 / 98 \\
5300 / 03\end{array}$ & 1FGL \\
\hline 1ES 1239+069 & 124148.3 & +063601 & $0.150^{4}$ & HBL & 1.9 & 26 & $4979 / 80$ & S96 \\
\hline PG 1246+586 & 124818.8 & +582029 & $>0.73:^{10}$ & $\mathrm{IBL}^{37}$ & 9.6 & 29 & $\begin{array}{c}5595 / 5601 / 03 / 05 \\
5621 / 24 / 25 / 29 \\
5630 / 31\end{array}$ & 1FGL \\
\hline 1ES $1255+244$ & 125731.9 & +241240 & $0.141^{27}$ & HBL & 26.0 & 16 & $\begin{array}{c}4531 / 34 / 68 / 80 \\
4581 / 82 / 83 / 84 \\
4585 / 86 / 87 / 91 \\
4907 / 11 / 23 / 50 \\
4970 / 79 / 80 \\
5207 / 08 / 36 \\
5591 / 5617 \\
5947 / 49 / 53 / 59 \\
5972 / 78 / 89 \\
6016 / 42 / 44 / 45 \\
6072 / 73 / 74\end{array}$ & SHBL, S96 \\
\hline BZB J1309+4305 & 130925.5 & +430506 & $0.691^{6}$ & HBL & 9.4 & 15 & $\begin{array}{c}5594 / 96 / 98 \\
5600 / 02 / 04 / 06 \\
5620 / 22 / 23 / 25\end{array}$ & 1FGL \\
\hline 1FGL J1323.1+2942 & 132302.4 & +294135 & - & $\mathrm{FSRQ}^{47}$ & 8.4 & 14 & $\begin{array}{c}4832 / 34 / 92 / 93 \\
4909 / 14 / 79 \\
5596 / 97 / 99 \\
5602 / 05 / 48 / 49\end{array}$ & 1FGL, ToO \\
\hline RX J1326.2+2933 & 132615.0 & +293331 & $0.431^{14}$ & HBL & 8.4 & 14 & Same as above & ROXA, C07 \\
\hline RGB J1341+399 & 134105.2 & +395946 & $0.169^{14}$ & HBL & 2.7 & 26 & $\begin{array}{c}4938 / 78 \\
5972 / 6045\end{array}$ & ROXA, N06 \\
\hline RGB J1351+112 & 135120.8 & +111453 & $>0.619^{28}$ & HBL & 6.2 & 24 & $\begin{array}{c}5210 / 21 / 39 / 40 \\
5241 / 43 / 46 \\
5293 / 97 / 98\end{array}$ & 1FGL \\
\hline RX J1353.4+5601 & 135328.1 & +530057 & $0.370^{14}$ & HBL & 2.5 & 26 & $5589 / 90 / 6002$ & ROXA, N06 \\
\hline RBS 1350 & 140659.2 & +164206 & $>0.623^{13}$ & HBL & 4.5 & 22 & $\begin{array}{c}5269 / 97 / 98 \\
5300 / 01\end{array}$ & 1FGL \\
\hline
\end{tabular}


Table 1 - Continued

\begin{tabular}{|c|c|c|c|c|c|c|c|c|}
\hline Source name & R.A. & Dec & $z^{a}$ & Type $^{b}$ & Exposure & $\hat{\theta}_{\text {zenith }}$ & MJDs & Selection $^{c}$ \\
\hline & [hr min sec] & [deg min sec] & & & [hr] & [deg] & {$[-50000]$} & \\
\hline RBS 1366 & 141756.7 & +254356 & $0.237^{12}$ & HBL & 10.0 & 17 & $\begin{array}{c}4591 / 92 / 93 / 94 \\
4596 / 99 \\
4611 / 12 / 13 / 15 / 16 \\
6046\end{array}$ & $\begin{array}{l}\text { SHBL, CG02 } \\
\text { ROXA }\end{array}$ \\
\hline 1ES $1421+582$ & 142238.9 & +580156 & $0.683^{14}$ & HBL & 3.4 & 28 & $\begin{array}{c}5324 / 25 / 26 / 28 / 30 \\
5333 / 35 / 50 / 51\end{array}$ & SHBL \\
\hline RGB J1439+395 & 143917.5 & +393243 & $0.344^{13}$ & HBL & 1.5 & 12 & $5620 / 6002$ & SHBL, ROXA \\
\hline 1RXS J144053.2+061013 & 144052.9 & +061016 & $0.396^{28}$ & $\mathrm{IBL}^{48}$ & 2.5 & 27 & $5731 / 32 / 34 / 35 / 36$ & 1FGL \\
\hline RBS 1452 & 150101.8 & +223806 & $0.235^{29}$ & $\mathrm{IBL}^{49}$ & 4.1 & 21 & $5297 / 98 / 99$ & 1FGL \\
\hline RGB J1532+302 & 153202.3 & +301629 & $0.065^{3}$ & HBL & 6.5 & 17 & $\begin{array}{l}4939 / 40 / 67 / 68 \\
4970 / 75 / 76 / 77\end{array}$ & $\mathrm{P} 00$ \\
\hline RGB J1533+189 & 153311.3 & +185429 & $0.305^{13}$ & HBL & 2.9 & 20 & $\begin{array}{c}5648 / 77 \\
5705 / 06 / 20\end{array}$ & $\begin{array}{c}\text { SHBL, ROXA } \\
\text { N06 }\end{array}$ \\
\hline 1ES $1533+535$ & 153500.9 & +532037 & $0.89:^{10}$ & HBL & 1.0 & 23 & $4256 / 6002$ & SHBL \\
\hline RGB J1610+671B & 161004.1 & +671026 & $0.067^{14}$ & HBL & 6.6 & 36 & $\begin{array}{c}4908 / 38 / 5268 \\
5292 / 93 / 94 / 95\end{array}$ & P00 \\
\hline 1ES $1627+402$ & 162901.3 & +400800 & $0.272^{3}$ & $\begin{array}{c}\text { HBL/ } \\
\text { NLS1 }^{50,51}\end{array}$ & 13.1 & 16 & $\begin{array}{c}4229 / 35 / 36 \\
4537 / 38 / 39 / 40 / 57 \\
4559 / 60 / 61 / 62 / 63 \\
4564 / 65 / 69 / 83 \\
4954\end{array}$ & $\mathrm{P} 02, \mathrm{~F} 04$ \\
\hline GB6 J1700+6830 & 170009.3 & +683007 & $0.301^{30}$ & $\mathrm{FSRQ}^{30}$ & 0.8 & 37 & $4914 / 16$ & 1FGL, ToO \\
\hline PKS $1717+177$ & 171913.0 & +174506 & $>0.58^{31}$ & $\mathrm{LBL}^{52}$ & 5.1 & 18 & $\begin{array}{c}4909 / 11 / 17 / 18 \\
4920 / 21 / 22\end{array}$ & 1FGL \\
\hline PKS $1725+045$ & 172825.0 & +042705 & $0.2966^{32}$ & FSRQ $^{53}$ & 0.3 & 27 & 4412 & M01 \\
\hline PKS 1749+096 & 175132.8 & +093901 & $0.32^{33}$ & $\mathrm{LBL}^{54}$ & 0.3 & 22 & 6072 & $\mathrm{ToO}$ \\
\hline RGB J1838+480 & 183949.2 & +480234 & $0.30:^{21}$ & $\mathrm{IBL}^{37}$ & 0.5 & 26 & 6090 & 2FGL \\
\hline RGB J1903+556 & 190311.6 & +554039 & $>0.58:^{7}$ & $\mathrm{IBL}^{4} \mathrm{~T}$ & 1.0 & 27 & 5099 & 1FGL \\
\hline 1FGL J1926.8+6153 & 192641.9 & +615441 & - & - & 1.3 & 31 & $5706 / 07$ & 1FGL \\
\hline PKS 2233-148 & 223634.1 & -143322 & $>0.49::^{34}$ & $\mathrm{LBL}^{55}$ & 0.3 & 49 & 6100 & $\mathrm{ToO}$ \\
\hline 3C 454.3 & 225357.7 & +160854 & $0.859^{35}$ & FSRQ $^{56}$ & 1.0 & 24 & $5504 / 31$ & $\mathrm{ToO}$ \\
\hline RGB J2322+346 & 232244.0 & +343614 & $0.098^{3}$ & $\mathrm{IBL}^{37}$ & 2.9 & 10 & $\begin{array}{l}4731 / 36 / 39 \\
4745 / 46 / 47\end{array}$ & P00 \\
\hline $1 \mathrm{ES} 2321+419$ & 232352.1 & +421059 & $>0.45^{36}$ & HBL & 4.2 & 21 & $\begin{array}{c}4773 / 76 \\
4802 / 03 / 30 / 31 \\
5091\end{array}$ & S96 \\
\hline B3 $2322+396$ & 232517.9 & +395737 & $>1.05^{32}$ & $\mathrm{LBL}^{54}$ & 1.0 & 16 & $5118 / 30$ & 1FGL \\
\hline 1FGL J2329.2+3755 & 232914.2 & +375415 & - & - & 3.3 & 11 & $\begin{array}{c}5470 / 71 / 72 / 75 / 76 \\
5477 / 78 / 80\end{array}$ & 1FGL \\
\hline 1RXS J234332.5+343957 & 234333.8 & +344004 & $0.366^{13}$ & HBL & 1.5 & 17 & 5912 & SHBL \\
\hline
\end{tabular}

${ }^{a}$ Unconstrained redshifts are indicated with a hyphen (-). If the redshift value is uncertain it is followed by a colon (:).

Redshift references: ${ }^{1}$ Fischer et al. (1998): ${ }^{2}$ Rau et al. (2012): ${ }^{2}$ Laurent-Muehleisen et al. (1998): ${ }^{4}$ Perlman et al. (1996): 5 Lawrence et al. (1986): ${ }^{\text {Shaw et al }}$ (2013); ${ }^{7}$ Meisner \& Romani (2010); ${ }^{2}$ Marcha et al. (1996); Böhringer et al. (2000); ${ }^{10}$ Sbarufatti et al. (2005); ${ }^{11}$ Cohen et al. (1987); ${ }^{12}$ this work; ${ }^{13}$ Piranomonte et al. (2007): ${ }^{14}$ Bauer et al. (2000):15 Lavaux \& Hudson (2011): ${ }^{16}$ Carswell et al. (1974): ${ }^{1 /}$ Falomo (1991): '18 Osterbrock \& Dahari (1983); ${ }^{19}$ Stickel et al. (1989); ${ }^{2}$ Plotkin et al. (2010); ${ }^{21}$ Nilsson et al. (2003); $; 2$ Stocke et al. (1991); ${ }^{2}$ Cao et al. (1999); ${ }^{24}$ Sbarufatti et al. (2009); ${ }^{25}$ Burbidge et al. (1977); ${ }^{22}$ White et al. (2000): ${ }^{2}$ Padovani \& Giommi (1995a): ${ }^{28}$ Sandrinelli et al. (2013): ${ }^{2}$ Jannuzi et al. (1993): ${ }^{3}$ Henstock et al. (1997); $;{ }^{3}$ Shaw et al. (2009); ${ }^{32}$ Eracleous \& Halpern (2004); ${ }^{3}$ Stickel et al. (1988); ${ }^{34}$ Sbarufatti et al. (2006); ${ }^{3}$ Smith et al. (1976); ${ }^{3}$ Falomo \& Kotilainen (1999).

${ }^{\mathrm{b}}$ Blazars of unknown type are indicated with a hyphen (-).

Blazar type references: ${ }^{37}$ Laurent-Muehleisen et al. (1999); ${ }^{38}$ Giommi et al. (2012); ${ }^{39}$ Chandra et al. (2012); ${ }^{40}$ Ackermann et al. (2012b); ${ }^{41}$ Nieppola et al. (2006); ${ }^{42}$ Abdo et al. (2009b); ${ }^{4}$ Massaro et al. (2012); ${ }^{4}$ Rani et al. (2011); ${ }^{45}$ Osterbrock \& Dahari (1983); ${ }^{4}$ Impey \& Neugebauer (1988); ${ }^{4 /}$ Cornwell et al.(1986); ${ }^{48}$ Ajello et al. (2014); ${ }^{49}$ Massaro et al. (2003); ${ }^{54}$ Padovani et al. (2002); ${ }^{51}$ Komossa et al. (2006); ${ }^{52}$ Li et al. (2010); ${ }^{53}$ Drinkwater et al. (1997); ${ }^{54}$ Abdo et al. (2010b); ${ }^{53}$ Lister et al. (2011); ;t Smith et al. (1976)

${ }^{c}$ Source selection references: $1 F G L$, Abdo et al. (2010a); $2 F G L$, Nolan et al. (2012); B97, Brinkmann et al. (1997); CG02, Costamante \& Ghisellini (2002); C07, Costamante (2007); F04, Falcone et al. (2004); M01, Mukherjee (2001); N06, Nieppola et al. (2006); ROXA, Turriziani et al. (2007); S96, Stecker et al. (1996); SHBL, Giommi et al. (2005); POO, Perlman (2000); PO2, Padovani et al. (2002); ToO, Target of Opportunity 
Table 2

List of known VHE sources observed but not detected by VERITAS in 2007-2012

\begin{tabular}{|c|c|c|c|c|c|c|c|c|c|}
\hline Source name & R.A. & Dec & $z^{a}$ & Type & Exposure & $\hat{\theta}_{\text {zenith }}$ & MJDs & Selection $^{b}$ & VHE detection $^{c}$ \\
\hline & {$[\mathrm{hr}$ min sec] } & [deg min sec] & & & [hr] & [deg] & {$[-50000]$} & & \\
\hline 1ES $0033+595$ & 003552.6 & +595005 & $0.086:^{1}$ & HBL & 22.6 & 31 & $\begin{array}{c}4411 / 18 / 19 / 20 \\
4421 / 38 / 40 / 48 \\
4464 / 66 / 76 \\
4734 / 36 / 37 / 74 \\
4775 / 76 / 77 \\
4803 / 04 / 06 \\
5866 / 67\end{array}$ & CG02, P00 & 1 \\
\hline RGB J0152+017 & 015233.5 & +014640 & $0.080^{2}$ & HBL & 8.4 & 35 & $\begin{array}{c}4421 / 22 / 37 / 38 \\
4439 / 40 / 47 / 48 \\
4449 / 50 / 64 / 65 / 78 \\
5537 / 5832 / 33 / 68\end{array}$ & CG02 & 2 \\
\hline RGB J0847+115 & 084713.0 & +113350 & $0.198^{3}$ & HBL & 12.1 & 25 & $\begin{array}{c}4499 / 4505 / 07 / 08 \\
4522 / 23 / 24 / 25 / 26 \\
5303 \\
5502 / 03 / 31 / 59\end{array}$ & SHBL & 3 \\
\hline RX J1136.5+6737 & 113630.1 & +673704 & $0.134^{4}$ & HBL & 7.7 & 37 & $\begin{array}{c}4860 / 61 / 91 / 92 \\
4918 / 21 \\
5292 / 94 / 99 \\
5303\end{array}$ & $\begin{array}{c}\text { CG02, SHBL } \\
\text { ROXA }\end{array}$ & 4 \\
\hline PKS $1222+216$ & 122454.4 & +212247 & $0.432^{5}$ & FSRQ & 25.8 & 16 & $\begin{array}{c}4939 / 5182 \\
5318 / 19 / 20 / 21 \\
5322 / 24 / 25 / 26 \\
5327 / 28 / 30 / 33 \\
5622 / 24 / 25 / 26 \\
5631 / 33 / 34\end{array}$ & $\mathrm{ToO}$ & $5 a, 5 b$ \\
\hline $3 \mathrm{C} 279$ & 125611.1 & -054722 & $0.536^{6}$ & FSRQ & 8.3 & 40 & $\begin{array}{c}5623 \\
5707 / 08 / 09 / 10 \\
5711 / 15 / 17 \\
5923 / 24 / 25 / 26 / 27 \\
6016\end{array}$ & $\mathrm{ToO}$ & $6 a, 6 b$ \\
\hline PKS 1510-089 & 151252.2 & -090622 & $0.361^{7}$ & FSRQ & 14.9 & 42 & $\begin{array}{c}4909 / 11 / 48 \\
5976 / 77 / 78 / 79 \\
5980 / 81 / 82 / 83 \\
5984 / 87\end{array}$ & $\mathrm{ToO}$ & $7 \mathrm{a}, 7 \mathrm{~b}$ \\
\hline RGB J1725+118 & 172504.3 & +115216 & $>0.35:^{8}$ & HBL & 10.0 & 23 & $\begin{array}{c}4593 / 94 \\
4615 / 16 / 17 / 18 \\
4619 / 20 / 21 / 22\end{array}$ & CG02 & 8 \\
\hline 0FGL J2001.0+4352 & 200113.5 & +435303 & $0.18:^{9}$ & HBL & 4.9 & 25 & $\begin{array}{c}5143 / 44 / 46 / 51 / 52 \\
5326 / 52 / 57\end{array}$ & 1FGL & 9 \\
\hline RGB J2243+203 & 224354.7 & +202104 & $>0.39:{ }^{10}$ & IBL & 4.1 & 17 & $\begin{array}{c}5094 / 98 / 99 \\
5101 / 16 / 28 / 29\end{array}$ & 1FGL & 10 \\
\hline B3 $2247+381$ & 225006.6 & +382558 & $0.118^{2}$ & HBL & 6.0 & 13 & $\begin{array}{c}5092 / 93 / 95 / 96 / 97 \\
5832 / 89\end{array}$ & 1FGL & 11 \\
\hline
\end{tabular}

${ }^{a}$ If the redshift value is uncertain it is followed by a colon (:). Redshift references: ${ }^{1}$ private communication from Perlman, see Falomo \& Kotilainen (1999); ${ }^{2}$ Laurent-Muehleisen et al.(1998); ${ }^{3}$ Cao et al. (1999); ${ }^{4}$ Bade et al. (1994); ${ }^{5}$ Burbidge \& Kinman (1966); ${ }^{6}$ Marziani et al. (1996); ${ }^{7}$ Thompson et al. (1990);

${ }^{8}$ Landoni et al. (2014); ${ }^{9}$ Aleksić et al. (2014a); ${ }^{10}$ Meisner \& Romani (2010)

b Selection references: see Table 1

${ }^{c}$ VHE detection references (see as well for the blazar subclass classification): ${ }^{1}$ Aleksić et al. (2015a); ${ }^{2}$ Aharonian et al. (2008a); ${ }^{3}$ Mirzoyan (2014c); ${ }^{4}$ Mirzoyan (2014a); ${ }^{5 a}$ Aleksić et al. (2011b); ${ }^{5 b}$ Holder (2014a); ${ }^{6 a}$ Albert et al. (2008); ${ }^{6 b}$ Aleksić et al. (2014c); ${ }^{\text {Ta }}$ Abramowski et al. (2013a); ${ }^{\text {b }}$ Aleksić et al. (2014b); ${ }^{8}$ Cortina (2013); ${ }^{9}$ Aleksić et al. (2014a); ${ }^{10}$ Holder (2014b); ${ }^{11}$ Aleksić et al. (2012)

Table 3

List of 2FGL sources in the VERITAS field of view of sources listed in Tables 1 and 2

\begin{tabular}{|c|c|c|c|c|c|c|}
\hline \hline Source name & Counterpart & R.A. $^{a}$ & Dec $^{a}$ & $z^{b}$ & Type $^{c}$ & in FoV of $^{d}$ \\
\hline & & {$[$ hr min sec] } & [deg min sec] & & & \\
\hline \hline 2FGL J0047.9+2232 & BWE 0045+2218 & 004802.5 & +223453 & $1.161^{1}$ & FSRQ $^{12}$ & RGB J0045+214 \\
2FGL J0148.6+0127 & PMN J0148+0129 & 014833.8 & +012901 & $0.940^{2}$ & - & RGB J0152+017 \\
2FGL J0158.4+0107 & - & 015825.4 & +010731 & - & - & RGB J0152+017 \\
2FGL J0205.4+3211 & 1Jy 0202+319 & 020504.9 & +321230 & $1.466^{3}$ & FSRQ $^{13}$ & B2 0200+30 \\
2FGL J0212.1+5318 & - & 021209.4 & +531819 & - & - & RGB J0214+517 \\
2FGL J0213.1+2245 & 1RXS J021252.2+224510 & 021252.8 & +224452 & $0.459^{2}$ & HBL $^{14}$ & RBS 0298 \\
2FGL J0326.1+2226 & TXS 0322+222 & 032536.8 & +222400 & $2.06^{4}$ & FSRQ $^{15}$ & RGB J0321+236 \\
2FGL J0440.4+1433 & TXS 0437+145 & 044021.1 & +143757 & - & - & 1RXS J044127.8+150455 \\
2FGL J0856.3+2058 & TXS 0853+211 & 085639.7 & +205743 & $>0.388^{5}$ & - & OJ 287 \\
2FGL J0929.5+5009 & QSO J0929+5013 & 092915.4 & +501336 & $0.370^{6}$ & IBL $^{16}$ & 1ES 0927+500 \\
\hline
\end{tabular}


Upper limits from five years of VERITAS blazar observations

Table 3 - Continued

\begin{tabular}{|c|c|c|c|c|c|c|}
\hline Source name & Counterpart & R.A. ${ }^{a}$ & $\operatorname{Dec}^{a}$ & $z^{b}$ & Type $^{c}$ & in $\mathrm{FoV}$ of ${ }^{d}$ \\
\hline & & [hr min sec] & [deg min sec] & & & \\
\hline 2FGL J1058.4+0133 & 4C 01.28 & 105829.6 & +013358 & $0.888^{7}$ & $\mathrm{FSRQ}^{13}$ & RBS 0921 \\
\hline 2FGL J1059.0+0222 & PMN J1059+0225 & 105906.0 & +022512 & - & - & RBS 0921 \\
\hline 2FGL J1141.0+6803 & 1RXS J114118.3+680433 & 114118.0 & +680433 & - & - & RX J1136.5+6737 \\
\hline 2FGL J1239.5+0728 & PKS $1236+077$ & 123824.6 & +073017 & $0.400^{8}$ & $\mathrm{FSRQ}^{17}$ & 1ES 1239+069 \\
\hline 2FGL J1245.1+5708 & GB6 J1245+5710 & 124510.0 & +570954 & $>0.521^{5}$ & $\mathrm{LBL}^{18}$ & PG $1246+586$ \\
\hline 2FGL J1303.1+2435 & VIPS J13030+2433 & 130303.2 & +243356 & $0.993^{9}$ & $\mathrm{LBL}^{19}$ & 1ES $1255+244$ \\
\hline 2FGL J1359.4+5541 & VIPS J13590+5544 & 135905.7 & +554429 & $1.014^{1}$ & $\mathrm{FSRQ}^{12}$ & RX J1353.4+5601 \\
\hline 2FGL J1722.7+1013 & TXS 1720+102 & 172244.6 & +101336 & $0.732^{10}$ & $\mathrm{FSRQ}^{20}$ & RGB J1725+118 \\
\hline 2FGL J1727.9+1220 & PKS $1725+123$ & 172807.1 & +121539 & $0.583^{11}$ & $\mathrm{FSRQ}^{20}$ & RGB J1725+118 \\
\hline 2FGL J1927.5+6117 & S4 1926+611 & 192730.4 & +61 1733 & - & $\mathrm{LBL}^{21}$ & 1FGL J1926.8+6153 \\
\hline 2FGL J1959.9+4212 & 1RXS J195956.1+421339 & 195956.1 & +421339 & - & - & 0FGL J2001.0+4352 \\
\hline
\end{tabular}

${ }^{a}$ Coordinates are provided for the counterpart. If the Fermi-LAT source is not associated with any lower-energy blazar, coordinates from the 2FGL catalog are given instead.

b Unconstrained redshifts are indicated with a hyphen (-).

Redshift references: ${ }^{1}$ Shaw et al. (2012); ${ }^{2}$ Shaw et al. (2013); ${ }^{3}$ Kraus \& Gearhart (1975); ${ }^{4}$ Halpern et al. (1986); 5 Plotkin et al. (2010); ${ }^{6}$ Healey et al. (2008); 7 Hewitt \& Burbidge (1993); ${ }^{8}$ White et al. (1988); ${ }^{9}$ Glikman et al. (2007); ${ }^{10}$ Afanas'Ev et al. (2005); ${ }^{11}$ Sowards-Emmerd et al. (2005).

c Blazars of unknown type are indicated with a hyphen $(-)$.

Blazar type references: ${ }^{12}$ Shaw et al. (2012); ${ }^{13}$ Kraus \& Gearhart (1975); ${ }^{14}$ Ajello et al. (2014); 15 Ghisellini et al. (2011); 16 Laurent-Muehleisen et al. (1999); ${ }^{17}$ Hewitt \& Burbidge (1993); ${ }^{8}$ Plotkin et al. (2010); ${ }^{9}$ Glikman et al. (2007); ${ }^{20}$ Afanas'Ev et al. (2005); ${ }^{21}$ Maselli et al. (2010).

d See Tables 1 and 2 for information on the exposure and the zenith angle of the observations.

The results presented in this paper have been obtained using a set of $\gamma$-hadron separation cuts specifically optimized for the detection of soft spectrum sources (differential spectrum parametrized by a power-law function $d N / d E=N\left(E / E_{0}\right)^{-\Gamma}$ with $\Gamma=3.5)$. The spectral index assumed is in line with the typical value of $\Gamma$ observed for VHE blazars (see Şentürk et al. 2013).

All the observations presented in this paper were made using the 'wobble' observing strategy (Fomin et al. 1994). Here, the telescopes point $0.5^{\circ}$ away from the target, alternatively in each of the four cardinal directions, to enable background estimation from the same field of view. This procedure ensures a similar acceptance for both the source (ON) and the background (OFF) regions. Regions overlapping bright stars are excluded from background estimates. The ratio of the $\mathrm{ON}$ over the OFF region size defines the background normalization parameter $\alpha$. The dead time of the telescope array is explicitly calculated and is approximately $10 \%$ for the observations described here. The exposure values provided in Table 1 are all corrected for dead time, and represent the effective live-time of VERITAS observations.

The VERITAS observations here have an average length of twenty minutes (referred to as a run), before switching targets or wobble directions. For quality assurance all the runs with a length lower than ten minutes were excluded, often being associated with technical problems, resulting in the early termination of the run. Additionally, all observations characterized by non-optimal weather conditions or malfunctioning hardware were excluded from the run selection. On certain occasions one of the VERITAS telescopes can be nonoperational due to technical problems; all the runs analyzed in this paper have at least three telescopes in operation. Runs with all four telescopes in operation represent the bulk (92\%) of the data.
In the standard configuration, VERITAS observations are not performed under bright-moonlight conditions (Moon illumination $>35 \%$ of full Moon). Since 2012, the VERITAS collaboration has started a new observing program in order to extend the duty cycle of the observatory and perform observations also under bright moonlight (Archambault et al. 2015). None of the data presented in this paper were taken under bright moonlight conditions. Observations performed under moderate moonlight (Moon illumination $<35 \%$ ) are included, and analyzed in the same manner as dark-time observations, with appropriate instrument response functions to account for the increased night-sky background.

The significance at the source location is computed using Equation 17 in $\mathrm{Li} \& \mathrm{Ma}(1983)$. The upper limit on the VHE flux is estimated according to Rolke et al. (2005) at the $99 \%$ confidence level. It is first calculated assuming three different values of the spectral index $(\Gamma=2.5,3.5$ and 4.5$)$ in order to estimate the decorrelation energy $E_{d e c}$ (the energy at which the upper limit estimate depends the least on the spectral index). The upper limit is then recomputed at the reference energy $E_{d e c}$ assuming a spectral index $\Gamma=3.5$. The threshold of the analysis (which depends mainly on the zenith angle of the observations) is also calculated. For every source we verified that, not only the overall significance is lower than 5 standard deviations $(\sigma)$, but that no flares have been detected, i.e. that none of the sources was detected at more than $4 \sigma$ during any single run.

For sources which are detected by Fermi-LAT (76\% of the sample), the flux is extrapolated into the VERITAS energy band, taking into account the absorption from the EBL using the model by Franceschini et al. (2008), which is in agreement with the most recent observational constraints (Abramowski et al. 2013b). The extrapolated flux is then compared to the VERITAS upper limit. If the VERITAS measurement is lower than the extrapolation, it means that 
an additional cut-off should be present in the $\gamma$-ray component between the Fermi-LAT and the VERITAS energy bands.

The results of the analysis are reported in Tables 4 and 5 (for known VHE sources) and 6 (for 2FGL sources in the VERITAS field of view). For every target, we provide the significance, the number of $\mathrm{ON}$ and OFF counts, the value of the $\alpha$ parameter (ratio of the ON over OFF region size), the threshold of the analysis $E_{t h}$, the decorrelation energy $E_{d e c}$, the differential flux upper limit at $E_{d e c}$, the integral flux upper limit (above $E_{t h}$, provided in Crab units, following Hillas et al. 1998 ${ }^{6}$ the ratio between the VERITAS differential upper limit and the extrapolation of the Fermi-LAT detection $\left(\Phi_{H E}\right.$, evaluated at $\left.E_{d e c}\right)$. Note that the values of the decorrelation and threshold energies are provided with three decimal values to ease any extrapolation to other energy bands, but they are known only to the second decimal value.

All the results presented in this paper have been crosschecked using a separate analysis, which provided consistent results for the single upper limit values, significance distributions (Section 3.2) and stacked analysis (Section 4).

\subsection{Notes on individual sources}

Among the blazars targeted by VERITAS between 2007 and 2012, eleven of them were later identified as VHE emitters. They are listed in Table 2. The VERITAS upper limits are useful in these cases to constrain the properties of the VHE emission during low-flux states, as well as the variability properties of the source. The discussion of these blazar observations, in order of R.A., follows:

- 1ES 0033+595 (HBL, $z=0.086:)$

VHE emission from 1ES 0033+595 was discovered by MAGIC (Aleksić et al. 2015a). The flux, measured from 24 hours of observations taken from August to October 2009, is $0.9 \%$ Crab above $290 \mathrm{GeV}^{7}$ The observed spectral index during the MAGIC observations is $3.8 \pm 0.7$. The VERITAS upper limit $(5.4 \% \mathrm{Crab}$ above $290 \mathrm{GeV}$ ) is fully consistent with the MAGIC measurement. The VHE variability of the source has been demonstrated by more recent VERITAS observations, which detected a bright VHE flare (with integral flux higher than $10 \%$ Crab) from the source during September 2013, with simultaneous X-ray and ultraviolet coverage by Swift (Benbow 2015). A paper presenting the results of this multi-wavelength campaign is currently in preparation.

- RGB J0152+017 (HBL, $z=0.08)$

This source has been known as a VHE emitter since

\footnotetext{
${ }^{6}$ The best-fit of the VHE emission from the Crab Nebula as measured with the Whipple 10-m telescope and presented in Hillas et al. (1998) is a power-law function with index $\Gamma=2.49$ and normalization $K=3.2 \times 10^{-11}$ $\mathrm{cm}^{-2} \mathrm{~s}^{-1} \mathrm{TeV}^{-1}$ at $1 \mathrm{TeV}$. The integral upper limits are computed from the differential ones and provided here as a reference. They can easily be recomputed for different values of $E_{t h}$, or for other definitions of the Crab unit. For example, using as a reference the MAGIC spectrum of the Crab nebula Aleksić et al.2015b, the Crab unit above $200 \mathrm{GeV}$ is $74 \%$ of the Whipple Crab unit above the same threshold.

${ }^{7}$ In order to ease the comparison between the results from different instruments, the integral fluxes provided in this section have been recomputed above the VERITAS threshold, when the spectral information is available, and are expressed in Crab units as defined in Hillas et al. (1998).
}

2008 (Aharonian et al. 2008a), when it was detected by H.E.S.S. at a flux of $2.6 \%$ Crab above $240 \mathrm{GeV}$, during 15 hours of observation. VERITAS observed the blazar in three different seasons: 2007-2008 (covering the H.E.S.S. period), 2010-2011 and 2011-2012. The VERITAS upper limit $(3.6 \%$ Crab above $240 \mathrm{GeV})$ is fully consistent with the H.E.S.S. detection.

- RGB J0847+115 (HBL, $z=0.198$ )

VHE emission from this blazar was announced by MAGIC in 2014, at a flux corresponding to $2.5 \% \mathrm{Crab}$ above $200 \mathrm{GeV}$ (Mirzoyan 2014c). No spectral information is currently available, but the MAGIC collaboration reported a preliminary classification of the source as an extreme-HBL, with synchrotron peak-frequency in hard-X-rays, and inverse-Compton peak-frequency at $\mathrm{TeV}$ energies. Evidence of VHE and optical variability was also claimed by MAGIC. The VERITAS upper limit $(2.0 \%$ of the Crab Nebula flux above $180 \mathrm{GeV})$ is marginally consistent with the preliminary flux estimate by MAGIC, and could be related to variability of the VHE emission.

- RX J1136.5+6737 (HBL, $z=0.134)$

The MAGIC collaboration recently reported the detection of this source at a flux of $1.5 \%$ Crab above 200 $\mathrm{GeV}$, in 20 hours of observations between January and April 2014 (Mirzoyan 2014a). No spectral information is available at the present time. The VERITAS upper limit $(5.2 \%$ Crab above $290 \mathrm{GeV})$ is fully consistent with the preliminary flux estimate by MAGIC.

- PKS 1222+216 (FSRQ, $z=0.432$ )

VHE emission from this FSRQ was detected by MAGIC in 2010 at a flux of the order of the Crab Nebula flux (Aleksić et al. 2011b). The detection of this VHE flare is of paramount importance for blazar physics: the rapid variability, and the fact that VHE photons can escape the bright photon field present in FSRQs was used to put constraints on the location of the $\gamma$-ray emitting region in blazars. The VERITAS non-detection constrains the low-flux state at a level of $2.2 \%$ Crab above $180 \mathrm{GeV}$.

During May 2014, PKS 1222+216 underwent another $\gamma$-ray flare, and VERITAS detected VHE emission at a flux of 3\% Crab (Holder 2014a). A paper describing the VERITAS detection in 2014 is currently in preparation.

- 3C 279 (FSRQ, $z=0.536)$

This quasar is the first of its class detected as a VHE emitter (Albert et al. 2008). VERITAS observations during 2011 were triggered by flaring activity observed at lower wavelengths (optical, X-rays and HE $\gamma$-rays). The same flare triggered observations with the MAGIC telescopes (Aleksić et al. 2014c), which resulted as well in no VHE detection (flux upper limit equal to $1.7 \%$ Crab above $260 \mathrm{GeV}$ ). The VERITAS flux upper limit $(2.1 \% \mathrm{Crab}$ above $260 \mathrm{GeV})$ is similar to the one measured with the MAGIC telescopes.

- PKS 1510-089 (FSRQ, $z=0.361$ )

Two VHE flares from this quasar have been reported so far: the first during March-April 2009, seen by H.E.S.S. (around $0.6 \%$ of the Crab Nebula flux above $260 \mathrm{GeV}$, 
see Abramowski et al.2013a), the second during February 2012, seen by MAGIC (around 1\% of the Crab Nebula flux above $260 \mathrm{GeV}$, see Aleksić et al. 2014b). The VERITAS observations presented in this paper are quasi-simultaneous with both flares (see Table 2 for details). For the 2009 flare, VERITAS observations were taken a few days before the VHE flare seen by H.E.S.S. For the 2012 flare, VERITAS observations were taken every night from February 19 to February 27, covering the Fermi-LAT flare. The VERITAS upper limit is $2.9 \%$ of the Crab Nebula flux above $260 \mathrm{GeV}$.

- RGB J1725+118 (HBL, $z>0.35$ )

The discovery of VHE emission from this blazar was recently reported by MAGIC (Cortina 2013) at a flux of $2 \%$ Crab above $140 \mathrm{GeV}$ during observations in May 2013 triggered by an elevated optical state. No spectral information is available at the present time. The VERITAS upper limit (3.2\% Crab above $200 \mathrm{GeV})$ is consistent with the MAGIC measurement.

- 0FGL J2001.0+4352 (HBL, $z=0.18$ :)

Early results from Fermi-LAT indicated that this source was a good candidate for IACTs, in particular due to its hard GeV spectrum (Abdo et al. 2009a). The MAGIC collaboration detected the source at a flux of $\sim 10 \%$ Crab during a single night (1.4 hours on July 16, 2010, see Aleksić et al. 2014a). The VERITAS upper limit clearly indicates that this blazar is variable at VHE, and that its baseline flux is below 5.2\% Crab above 200 $\mathrm{GeV}$.

- RGB J2243+203 (HBL, $z>0.39$ )

VHE emission from this source was detected by VERITAS during December 2014, following a trigger from a high Fermi-LAT flux (Holder 2014b; Abeysekara 2015). Preliminary analysis indicates that the flux from the flaring blazar was at $\sim 4 \%$ Crab above $180 \mathrm{GeV}$. The upper limits computed from 2009 observations indicate that the VHE emission from this source is variable, being significantly lower $(2.1 \%$ Crab above 170 $\mathrm{GeV}$ ) than the 2014 detection.

- B3 2247+381 (HBL, $z=0.119)$

This source was detected by MAGIC in 14 hours of observations from September to October 2010, at a flux of $2.2 \%$ Crab above $170 \mathrm{GeV}$ (Aleksić et al. 2012). The MAGIC observations were triggered by a high optical state, and there is evidence of variability in the simultaneous X-ray light curve. VERITAS observations do not cover the MAGIC detection, nor the high optical flux state measured by the Tuorla observatory. The non-detection by VERITAS (flux upper limit equal to $1.8 \%$ Crab above $170 \mathrm{GeV}$ ) constrains the low-state flux of this blazar to be lower than the MAGIC detection, suggesting that it may have been related to a VHE high-flux state.

In addition to these known VHE emitters, we discuss a few other targets with noteworthy histories:

- 1ES 0037+405 (HBL, $z$ unknown)

VERITAS observations of this target were taken as a self-triggered ToO. During observations of the Andromeda galaxy (M31, see Bird 2015), a $4 \sigma$ hotspot coincident with this blazar was observed in the reconstructed sky-map. However, further observations did not confirm the hotspot, and the cumulative significance is $1.5 \sigma$ in 36 hours.

- OJ 287 (LBL, $z=0.306)$

This blazar is one of the most studied objects of its kind due to a clear periodicity in its optical lightcurve, with a period of about twelve years. The VERITAS observations presented in this work cover the last active phase in Fall 2007 (from December 4, 2007 to January 1, 2008), with additional observations during 2010. The VHE upper limits are comparable to the ones measured with the MAGIC telescopes and presented by Seta et al. (2009).

- 1FGL J1323.1+2942 (FSRQ, $z$ unknown) and RX J1326.2+2933 (HBL, $z=0.431$ )

Although the angular distance between the two sources is only $43^{\prime}$, they are not the same blazar. VERITAS can resolve the two objects, and they have been targeted by VERITAS independently (see last column of Table 1). Since they are well within the VERITAS FoV, the exposures on these two objects have been merged into a single dataset.

- B2 0912+29 (HBL, $z=0.36)$

This blazar shows the highest significance in our dataset ( $3.5 \sigma$ in 11.7 hours). This excess was confirmed at the same significance level by the cross-check analysis chain. Further observations were taken during the 2013 and 2014 observing seasons, but the initial excess did not increase. While VHE blazars are known to be variable, and one could interpret the lack of a detection in 2013-2014 as due to variability, we also note that the probability of a $3.5 \sigma$ excess reduces to only $2.0 \sigma$ when 103 trials (the sources from Tables 1 and 3 are taken into account, and it is thus not enough to make any claim.

\subsection{Significance distributions}

In Fig. 1 we present the distribution of the significances for all the sources presented in our work. Given that the blazar population at VHE is not homogeneous (see the Introduction), and depends on both the blazar sub-class (which is correlated with the energy of the high-energy SED peak) and the blazar redshift (which implies a different level of EBL absorption), significance distributions are produced as a function of these two parameters. For the redshift division (left plot of Fig. 1), redshifts lower or higher than 0.6 were considered, along with unknown redshift. Concerning the division of blazar sub-classes (right plot of Fig. 1), the sources were categorized as HBLs, IBLs/LBLs/FSRQs, and blazars of unknown type. The Gaussian distribution expected from a sample with average $\hat{X}=0$ and $\sigma=1$ is overlaid on the significance distribution. A fit of the histogram with a Gaussian function instead yields $\hat{X}=0.3 \pm 0.1$ and $\sigma=1.2 \pm 0.1$. 
Table 4

Analysis results and flux upper limits for the non-detected AGN observed by VERITAS

\begin{tabular}{|c|c|c|c|c|c|c|c|c|c|}
\hline Source name & $\sigma$ & $\mathrm{ON}$ & OFF & $\alpha$ & $\begin{array}{c}\mathrm{E}_{\mathrm{th}} \\
{[\mathrm{TeV}]}\end{array}$ & $\begin{array}{l}\mathrm{E}_{\mathrm{dec}} \\
{[\mathrm{TeV}]}\end{array}$ & $\begin{array}{c}\mathrm{UL}_{\Theta} E_{\mathrm{dec}} \\
{\left[10^{-12} \mathrm{~cm}^{-2} \mathrm{~s}^{-1} \mathrm{TeV}^{-1}\right]}\end{array}$ & $\begin{array}{l}\mathrm{UL}>E_{\mathrm{th}} \\
{[\% \text { C.U.] }}\end{array}$ & $\mathrm{UL} / \Phi_{H E}$ \\
\hline RBS 0042 & 0.02 & 1239 & 6455 & 0.192 & 0.182 & 0.345 & 8.7 & 2.2 & $0.2(\mathrm{z}=0.1)$ \\
\hline RBS 0082 & 0.16 & 1680 & 9062 & 0.185 & 0.166 & 0.264 & 16.9 & 1.8 & 45.0 \\
\hline 1ES $0037+405$ & 1.50 & 7515 & 64132 & 0.115 & 0.166 & 0.322 & 29.3 & 6.3 & $\ldots$ \\
\hline 1RXS J0045.3+2127 & 2.04 & 224 & 1116 & 0.172 & 0.166 & 0.297 & 54.6 & 8.9 & $3.0 / 14.8(\mathrm{z}=0.1 / 0.5)$ \\
\hline RGB J0110+418 & -0.02 & 801 & 4810 & 0.167 & 0.182 & 0.299 & 22.4 & 3.4 & $\ldots$ \\
\hline 1ES 0120+340 & 1.47 & 1174 & 5215 & 0.214 & 0.166 & 0.283 & 24.6 & 3.4 & 1.4 \\
\hline QSO $0133+476$ & 1.24 & 114 & 496 & 0.202 & 0.417 & 0.728 & 11.8 & 17.4 & $1.9 \mathrm{e} 4$ \\
\hline B2 $0200+30$ & 1.38 & 495 & 2775 & 0.167 & 0.151 & 0.273 & 51.9 & 6.9 & 59.6 \\
\hline CGRaBS J0211+1051 & 1.01 & 977 & 5659 & 0.167 & 0.200 & 0.318 & 20.8 & 3.6 & 3.4 \\
\hline RGB J0214+517 & 0.33 & 1113 & 5877 & 0.187 & 0.182 & 0.336 & 15.9 & 3.7 & $\ldots$ \\
\hline RBS 0298 & 1.76 & 606 & 3245 & 0.173 & 0.240 & 0.435 & 21.5 & 9.2 & $\ldots$ \\
\hline RBS 0319 & -0.52 & 61 & 393 & 0.167 & 0.219 & 0.351 & 38.4 & 8.6 & 44.9 \\
\hline $\mathrm{AO} 0235+16$ & 0.63 & 704 & 4116 & 0.167 & 0.182 & 0.311 & 18.7 & 3.2 & 9.6 \\
\hline RGB J0250+172 & -0.06 & 1274 & 5637 & 0.226 & 0.166 & 0.316 & 13.5 & 2.7 & 1.4 \\
\hline 2FGL J0312.8+2013 & -0.36 & 2124 & 9046 & 0.238 & 0.166 & 0.257 & 10.0 & 1.0 & $0.5 / 0.6(\mathrm{z}=0.1 / 0.5)$ \\
\hline RGB J0314+247 & 1.07 & 691 & 3967 & 0.167 & 0.240 & 0.460 & 16.4 & 8.5 & $\ldots$ \\
\hline RGB J0314+063 & 1.18 & 76 & 326 & 0.200 & 0.182 & 0.294 & 76.7 & 11.0 & $\ldots$ \\
\hline RGB J0321+236 & 1.24 & 3065 & 17948 & 0.167 & 0.138 & 0.233 & 31.2 & 2.6 & $2.0 / 2.9(\mathrm{z}=0.1 / 0.5)$ \\
\hline B2 $0321+33$ & -0.03 & 2190 & 8223 & 0.267 & 0.166 & 0.272 & 9.7 & 1.2 & 15.9 \\
\hline 1FGL J0333.7+2919 & 0.37 & 158 & 606 & 0.223 & 0.138 & 0.226 & 101.0 & 7.6 & $2.7 / 7.4(\mathrm{z}=0.1 / 0.5)$ \\
\hline 1RXS J044127.8+150455 & 1.83 & 2351 & 10636 & 0.212 & 0.182 & 0.338 & 17.5 & 4.1 & ... \\
\hline 2FGL J0423.3+5612 & 0.67 & 283 & 1522 & 0.178 & 0.240 & 0.457 & 16.4 & 8.3 & $1.9 / 32.6(\mathrm{z}=0.1 / 0.5)$ \\
\hline 1FGL J0423.8+4148 & -0.22 & 240 & 1462 & 0.167 & 0.166 & 0.274 & 46.4 & 5.7 & $0.6 / 2.2(\mathrm{z}=0.1 / 0.5)$ \\
\hline 1ES 0446+449 & -1.49 & 1482 & 10866 & 0.142 & 0.219 & 0.363 & 4.7 & 1.2 & $\ldots$ \\
\hline RGB J0505+612 & -1.50 & 2167 & 9896 & 0.227 & 0.219 & 0.377 & 4.1 & 1.2 & $0.6 / 5.7(\mathrm{z}=0.1 / 0.5)$ \\
\hline 1FGL J0515.9+1528 & -0.54 & 1149 & 6734 & 0.173 & 0.151 & 0.283 & 16.7 & 2.5 & $0.9 / 3.8(\mathrm{z}=0.1 / 0.5)$ \\
\hline 2FGL J0540.4+5822 & 0.43 & 226 & 1315 & 0.167 & 0.240 & 0.459 & 16.5 & 8.5 & $2.8 / 49.4(\mathrm{z}=0.1 / 0.5)$ \\
\hline RGB J0643+422 & 0.46 & 240 & 1282 & 0.181 & 0.200 & 0.369 & 32.7 & 9.5 & $\ldots$ \\
\hline RGB J0656+426 & 1.16 & 1960 & 9607 & 0.198 & 0.200 & 0.322 & 20.2 & 3.6 & $\ldots$ \\
\hline 1ES 0735+178 & -1.20 & 1259 & 6865 & 0.190 & 0.166 & 0.260 & 9.0 & 0.9 & 0.5 \\
\hline BZB J0809+3455 & -0.24 & 252 & 1537 & 0.167 & 0.151 & 0.251 & 39.8 & 4.0 & $\ldots$ \\
\hline PKS 0829+046 & -0.77 & 465 & 2465 & 0.196 & 0.240 & 0.379 & 10.1 & 2.7 & 0.6 \\
\hline Mrk 1218 & 2.44 & 1589 & 8994 & 0.165 & 0.166 & 0.280 & 43.1 & 5.7 & $\ldots$ \\
\hline OJ 287 & 0.97 & 2197 & 12966 & 0.166 & 0.182 & 0.296 & 17.4 & 2.6 & 3.1 \\
\hline B2 $0912+29$ & 3.49 & 3466 & 19492 & 0.167 & 0.138 & 0.228 & 45.9 & 3.6 & 1.6 \\
\hline 1ES $0927+500$ & -0.18 & 2378 & 11404 & 0.209 & 0.182 & 0.346 & 11.0 & 2.8 & $\ldots$ \\
\hline RBS 0831 & -0.31 & 394 & 2403 & 0.167 & 0.166 & 0.297 & 29.5 & 4.8 & $\ldots$ \\
\hline RGB J1012+424 & 0.18 & 270 & 1324 & 0.167 & 0.219 & 0.316 & 43.5 & 6.7 & 22.2 \\
\hline 1ES $1028+511$ & 1.16 & 4610 & 27154 & 0.167 & 0.182 & 0.305 & 12.4 & 2.0 & 1.4 \\
\hline RGB J1037+571 & -1.53 & 790 & 3798 & 0.221 & 0.200 & 0.331 & 5.7 & 1.1 & $2.5(\mathrm{z}=0.6)$ \\
\hline RGB J1053+494 & -0.76 & 1397 & 8567 & 0.167 & 0.200 & 0.386 & 6.4 & 2.2 & 0.5 \\
\hline RBS 0921 & 0.84 & 633 & 3534 & 0.173 & 0.240 & 0.354 & 20.1 & 4.2 & $\ldots$ \\
\hline RBS 0929 & -0.62 & 923 & 4678 & 0.202 & 0.166 & 0.319 & 11.3 & 2.4 & $0.5 / 2.7(\mathrm{z}=0.1 / 0.5)$ \\
\hline 1ES 1106+244 & -1.65 & 200 & 1151 & 0.197 & 0.151 & 0.257 & 14.3 & 1.5 & 3.3 \\
\hline RX J1117.1+2014 & 0.16 & 2545 & 12950 & 0.190 & 0.151 & 0.281 & 12.5 & 1.8 & 0.18 \\
\hline 1ES $1118+424$ & 0.39 & 1685 & 9703 & 0.172 & 0.151 & 0.267 & 22.4 & 2.8 & 0.39 \\
\hline S4 1150+497 & -0.53 & 749 & 4589 & 0.167 & 0.182 & 0.315 & 12.9 & 2.3 & 70.0 \\
\hline RGB J1231+287 & 0.74 & 1258 & 6664 & 0.185 & 0.138 & 0.243 & 36.6 & 3.5 & 12.2 \\
\hline 1ES 1239+069 & -0.86 & 224 & 1429 & 0.167 & 0.240 & 0.369 & 8.7 & 2.1 & $\ldots$ \\
\hline PG $1246+586$ & 0.23 & 2123 & 12670 & 0.167 & 0.200 & 0.363 & 9.5 & 2.4 & $14.5(\mathrm{z}=0.73)$ \\
\hline 1ES $1255+244$ & 2.24 & 5127 & 29732 & 0.167 & 0.166 & 0.315 & 12.4 & 2.5 & $\ldots$ \\
\hline BZB J1309+4305 & 0.54 & 2359 & 14020 & 0.167 & 0.151 & 0.298 & 17.8 & 3.2 & 7.9 \\
\hline 1FGL J1323.1+2942 & 0.24 & 1781 & 14622 & 0.121 & 0.151 & 0.243 & 18.0 & 1.6 & $1.2 / 3.7(\mathrm{z}=0.1 / 0.5)$ \\
\hline RX J1326.2+2933 & 1.36 & 1771 & 14150 & 0.127 & 0.166 & 0.256 & 17.8 & 1.7 & $\ldots$ \\
\hline RGB J1341+399 & 0.00 & 381 & 2286 & 0.167 & 0.200 & 0.405 & 12.7 & 5.1 & $\ldots$ \\
\hline RGB J1351+112 & 1.44 & 1715 & 8994 & 0.184 & 0.151 & 0.248 & 30.0 & 2.8 & $2.9(\mathrm{z}=0.62)$ \\
\hline RX J1353.4+5601 & 0.65 & 569 & 3163 & 0.175 & 0.200 & 0.339 & 24.8 & 5.3 & $\ldots$ \\
\hline RBS 1350 & 0.55 & 1387 & 8190 & 0.167 & 0.151 & 0.248 & 27.9 & 2.6 & $\ldots$ \\
\hline RBS 1366 & 1.89 & 1789 & 9843 & 0.173 & 0.200 & 0.327 & 17.1 & 3.3 & 7.9 \\
\hline 1ES $1421+582$ & 0.17 & 674 & 4016 & 0.167 & 0.219 & 0.378 & 13.2 & 3.8 & \\
\hline RGB J1439+395 & 0.80 & 404 & 2321 & 0.167 & 0.151 & 0.246 & 62.4 & 5.7 & 5.0 \\
\hline 1RXS J144053.2+061013 & -0.09 & 424 & 2556 & 0.167 & 0.200 & 0.343 & 13.8 & 3.1 & 16.7 \\
\hline RBS 1452 & 0.03 & 1232 & 7474 & 0.165 & 0.151 & 0.254 & 25.9 & 2.7 & 0.5 \\
\hline RGB J1532+302 & -0.56 & 812 & 3648 & 0.227 & 0.166 & 0.348 & 10.7 & 3.0 & $\ldots$ \\
\hline RGB J1533+189 & -1.44 & 653 & 4460 & 0.167 & 0.151 & 0.293 & 8.5 & 1.5 & $\ldots$ \\
\hline 1ES $1533+535$ & 0.54 & 191 & 973 & 0.188 & 0.182 & 0.324 & 41.7 & 8.9 & $\ldots$ \\
\hline RGB J1610+671B & -1.75 & 1318 & 6510 & 0.214 & 0.263 & 0.516 & 1.6 & 1.1 & $\cdots$ \\
\hline 1ES 1627+402 & -0.33 & 2191 & 13244 & 0.167 & 0.182 & 0.360 & 7.3 & 2.1 & $\ldots$ \\
\hline GB6 J1700+6830 & -1.98 & 81 & 610 & 0.167 & 0.316 & 0.528 & 3.5 & 2.2 & 161 \\
\hline PKS 1717+177 & 0.42 & 934 & 4343 & 0.212 & 0.182 & 0.290 & 19.3 & 2.6 & $3.1(\mathrm{z}=0.58)$ \\
\hline PKS $1725+045$ & -0.58 & 41 & 271 & 0.167 & 0.200 & 0.329 & 37.5 & 7.3 & 127 \\
\hline PKS 1749+096 & -1.00 & 64 & 438 & 0.167 & 0.166 & 0.257 & 43.6 & 4.3 & 1.6 \\
\hline RGB J1838+480 & 0.27 & 87 & 329 & 0.167 & 0.200 & 0.346 & 52.3 & 12.1 & 4.2 \\
\hline RGB J1903+556 & 0.19 & 164 & 968 & 0.167 & 0.240 & 0.398 & 22.4 & 7.0 & $22.2(\mathrm{z}=0.58)$ \\
\hline
\end{tabular}


Table 4 - Continued

\begin{tabular}{|c||c|c|c|c||c|c|c|c|c|}
\hline \hline Source name & $\sigma$ & ON & OFF & $\alpha$ & $\begin{array}{c}\mathrm{E}_{\mathrm{th}} \\
{[\mathrm{TeV}]}\end{array}$ & $\begin{array}{c}\mathrm{E}_{\mathrm{dec}} \\
{[\mathrm{TeV}]}\end{array}$ & $\begin{array}{c}\mathrm{UL}_{\varrho} @ E_{\mathrm{dec}} \\
{\left[10^{-12} \mathrm{~cm}^{-2} \mathrm{~s}^{-1} \mathrm{TeV}^{-1}\right]}\end{array}$ & $\begin{array}{c}\mathrm{UL}_{>E_{\mathrm{th}}} \\
{[\% \text { C.U. }}\end{array}$ & UL/ $\Phi_{H E}$ \\
\hline 1FGL J1926.8+6153 & 0.62 & 231 & 1326 & 0.167 & 0.240 & 0.408 & 21.6 & 7.4 & $1.1 / 12.5(\mathrm{z}=0.1 / 0.5)$ \\
PKS 2233-148 & 0.06 & 32 & 190 & 0.167 & 0.501 & 0.829 & 7.8 & 15.0 & $1.4 \mathrm{e} 3(\mathrm{z}=0.49)$ \\
3C 454.3 & -1.21 & 220 & 981 & 0.25 & 0.138 & 0.250 & 23.0 & 2.5 & 0.6 \\
RGB J2322+346 & -0.42 & 518 & 2926 & 0.181 & 0.182 & 0.296 & 19.2 & 2.8 & 1.4 \\
1ES 2321+419 & 2.05 & 992 & 3686 & 0.250 & 0.219 & 0.414 & 23.7 & 9.4 & $15.9(\mathrm{z}=0.5)$ \\
B3 2322+396 & -0.63 & 131 & 705 & 0.197 & 0.166 & 0.256 & 49.2 & 4.8 & $80(\mathrm{z}=1.05)$ \\
1FGL J2329.2+3755 & -0.13 & 847 & 5106 & 0.167 & 0.166 & 0.254 & 27.3 & 2.6 & $1.0 / 3.4(\mathrm{z}=0.1 / 0.5)$ \\
1RXS J234332.5+343957 & 0.80 & 341 & 1952 & 0.167 & 0.151 & 0.250 & 53.5 & 5.2 & 3.2 \\
\hline
\end{tabular}

Table 5

Results and upper limits for the known VHE sources

\begin{tabular}{|c||c|c|c|c||c|c|c|c|c|}
\hline \hline Source name & $\sigma$ & ON & OFF & $\alpha$ & $\begin{array}{c}\mathrm{E}_{\mathrm{th}} \\
{[\mathrm{TeV}]}\end{array}$ & $\begin{array}{c}\mathrm{E}_{\mathrm{dec}} \\
{[\mathrm{TeV}]}\end{array}$ & $\begin{array}{c}\mathrm{UL}_{\mathrm{O}} E_{\mathrm{dec}} \\
{\left[10^{-12} \mathrm{~cm}^{-2} \mathrm{~s}^{-1} \mathrm{TeV}^{-1}\right]}\end{array}$ & $\begin{array}{c}\mathrm{UL}>E_{\mathrm{th}} \\
{[\% \text { C.U. }]}\end{array}$ & $\mathrm{UL} / \Phi_{H E}$ \\
\hline \hline 1ES 0033+595 & 3.23 & 4560 & 20321 & 0.214 & 0.288 & 0.490 & 10.0 & 5.4 & $0.8(\mathrm{z}=0.086)$ \\
RGB J0152+017 & 1.83 & 1720 & 8693 & 0.188 & 0.240 & 0.376 & 14.1 & 3.6 & 1.2 \\
RGB J0847+115 & -0.03 & 3391 & 19889 & 0.171 & 0.182 & 0.338 & 8.63 & 2.0 & 0.3 \\
RX J1136.5+6737 & 1.10 & 1324 & 7271 & 0.177 & 0.288 & 0.519 & 7.86 & 5.2 & 1.3 \\
PKS 1222+216 & 3.40 & 7482 & 39770 & 0.180 & 0.182 & 0.257 & 24.1 & 2.2 & 0.2 \\
3C 279 & 0.10 & 1479 & 8849 & 0.167 & 0.263 & 0.430 & 5.5 & 2.1 & 2.6 \\
PKS 1510-089 & 2.00 & 2691 & 13698 & 0.167 & 0.263 & 0.496 & 4.7 & 2.9 & 1.1 \\
RGB J1725+118 & 2.29 & 1979 & 10833 & 0.079 & 0.200 & 0.316 & 18.7 & 3.2 & $0.6 / 2.6(\mathrm{z}=0.1 / 0.5)$ \\
0FGL J2001.0+4352 & 0.76 & 1102 & 6449 & 0.167 & 0.200 & 0.384 & 15.5 & 5.2 & $0.3(\mathrm{z}=0.2)$ \\
RGB J2243+203 & -0.12 & 1111 & 6458 & 0.173 & 0.166 & 0.263 & 19.3 & 2.1 & $0.3(\mathrm{z}=0.39)$ \\
B3 2247+381 & -0.93 & 1447 & 8042 & 0.185 & 0.166 & 0.315 & 8.8 & 1.8 & 0.5 \\
\hline
\end{tabular}

Table 6

Results and upper limits for the 2FGL sources in the VERITAS field of view

\begin{tabular}{|c||c|c|c|c||c|c|c|c|c|}
\hline \hline Source name & $\sigma$ & ON & OFF & $\alpha$ & $\begin{array}{c}E_{\text {th }} \\
{[\mathrm{TeV}]}\end{array}$ & $\begin{array}{c}\mathrm{E}_{\mathrm{dec}} \\
{[\mathrm{TeV}]}\end{array}$ & $\begin{array}{c}\mathrm{UL}_{\varrho} E_{\mathrm{dec}} \\
{\left[10^{-12} \mathrm{~cm}^{-2} \mathrm{~s}^{-1} \mathrm{TeV}^{-1}\right]}\end{array}$ & $\begin{array}{c}\mathrm{UL}>E_{\text {th }} \\
{[\% \text { C.U.] }}\end{array}$ & $\mathrm{UL/} \Phi_{H E}$ \\
\hline 2FGL J0047.9+2232 & 0.32 & 123 & 2058 & 0.0770 & 0.200 & 0.314 & 70.5 & 11.6 & $1.6 \mathrm{e} 4$ \\
2FGL J0148.6+0127 & 1.15 & 1239 & 17272 & 0.063 & 0.240 & 0.412 & 25.1 & 8.9 & $6.7 \mathrm{e} 3$ \\
2FGL J0158.4+0107 & -1.62 & 582 & 13194 & 0.0625 & 0.240 & 0.435 & 16.6 & 7.1 & $150 / 2.2 \mathrm{e} 3(\mathrm{z}=0.1 / 0.5)$ \\
2FGL J0205.4+3211 & 1.76 & 232 & 3743 & 0.0524 & 0.166 & 0.290 & 102 & 15.4 & $5.4 \mathrm{e} 5$ \\
2FGL J0212.1+5318 & -1.22 & 389 & 8089 & 0.0512 & 0.200 & 0.377 & 37.8 & 11.9 & $1.0 / 9.1(\mathrm{z}=0.1 / 0.5)$ \\
2FGL J0213.1+2245 & 0.57 & 501 & 7460 & 0.0655 & 0.182 & 0.295 & 63.3 & 9.2 & 29.3 \\
2FGL J0326.1+2226 & 0.22 & 1342 & 25891 & 0.0515 & 0.151 & 0.249 & 92.9 & 9.0 & $2.5 \mathrm{e} 4$ \\
2FGL J0440.4+1433 & 0.20 & 1028 & 19458 & 0.0525 & 0.182 & 0.340 & 50.3 & 12.1 & $60 / 416(\mathrm{z}=0.1 / 0.5)$ \\
2FGL J0856.3+2058 & 0.19 & 1686 & 21961 & 0.0764 & 0.182 & 0.297 & 37.1 & 5.6 & $53(\mathrm{z}=0.5)$ \\
2FGL J0929.5+5009 & 3.28 & 1712 & 17578 & 0.0884 & 0.200 & 0.349 & 40.2 & 9.6 & 28 \\
2FGL J1058.4+0133 & 1.19 & 319 & 5882 & 0.0506 & 0.240 & 0.373 & 51.7 & 12.9 & 985 \\
2FGL J1059.0+0222 & -0.37 & 533 & 7097 & 0.0764 & 0.240 & 0.359 & 25.8 & 5.7 & $30 / 240(\mathrm{z}=0.1 / 0.5)$ \\
2FGL J1141.0+6803 & 0.17 & 1195 & 12124 & 0.0981 & 0.288 & 0.525 & 7.5 & 5.1 & $0.7 / 18.9(\mathrm{z}=0.1 / 0.5)$ \\
2FGL J1239.5+0728 & 1.41 & 198 & 2766 & 0.0644 & 0.240 & 0.368 & 55.1 & 13.1 & 67 \\
2FGL J1245.1+5708 & 2.34 & 1418 & 24171 & 0.0545 & 0.219 & 0.369 & 40.7 & 10.8 & $276(\mathrm{z}=0.52)$ \\
2FGL J1303.1+2435 & 0.33 & 3021 & 57951 & 0.0518 & 0.166 & 0.322 & 36.2 & 7.8 & 117 \\
2FGL J1359.4+5541 & 1.74 & 507 & 5493 & 0.0850 & 0.219 & 0.347 & 50.5 & 10.8 & $1.4 \mathrm{e} 5$ \\
2FGL J1722.7+1013 & 1.73 & 693 & 14010 & 0.0462 & 0.200 & 0.332 & 72.2 & 14.5 & 433 \\
2FGL J1727.9+1220 & -0.56 & 1816 & 23431 & 0.0789 & 0.200 & 0.315 & 15.3 & 2.5 & 41 \\
2FGL J1927.5+6117 & -1.94 & 127 & 1964 & 0.077 & 0.240 & 0.412 & 12.1 & 4.3 & $5.1 / 62(\mathrm{z}=0.1 / 0.5)$ \\
2FGL J1959.9+4212 & 1.82 & 458 & 9118 & 0.037 & 0.219 & 0.347 & 64.4 & 13.8 & $24 / 176(\mathrm{z}=0.1 / 0.5)$ \\
\hline
\end{tabular}




\section{STACKED ANALYSIS}

Motivated by the skew in the significance distribution and in order to study if there is any evidence of emission from a population of blazars below the VERITAS sensitivity level, a stacked analysis of the data-set is performed. For every source the $\gamma$-ray excess (ON - $\alpha$ OFF) and its uncertainty (the excess divided by the significance) are calculated. We then compute the sum of the excesses, and its uncertainty (the square root of the sum of the squared uncertainties), whose ratio provides the significance of the stacked excess. Sources known as VHE emitters are excluded from the stacked analysis, which only includes sources listed in Tables 1 and 3 .

The stacked analysis indicates that there is evidence of VHE emission at a level of $4.0 \sigma$, corresponding to an excess of $1990 \gamma$-rays. The same study is then performed for sub-samples of the overall data-set. The majority of the excess $(3.0 \sigma)$ comes from nearby $(z<0.6)$ HBLs. On the other hand, the stacked analysis including only non-HBL sources located at an unknown distance or $z>0.6$ results in a stacked significance of $1.1 \sigma$. However, because nearby HBLs are considered the most likely VHE candidates, there are more of them and they often have deeper exposures. So this study has more sensitivity to the nearby HBLs. Indeed, the VERITAS exposure on HBLs located at $z<0.6$ is about 196 hours. By assuming that the $4.0 \sigma$ stacked excess comes from a constant signal from all sources in 570 hours, one would expect a $2.3 \sigma$ excess in 196 hours. The excess from the $z<0.6 \mathrm{HBLs}$ is thus compatible with this expectation, and it is not possible to claim that the stacked excess is dominated by a particular blazar population.

The MAGIC collaboration has also reported evidence for VHE emission from a stacked sample of IBL/HBL sources (Aleksić et al. 2011a), detecting a signal at a significance level of $4.9 \sigma$ from an exposure of 394 hours. The following sources included in the present work are also part of the MAGIC sample: 1ES 0120+340, 1RXS J044127.8+150455, 1ES 0927+500, 1ES 1028+511, RX J1117.1+2014, RX 1136.5+6737, and RBS 1366. The four sources with the highest significance in the MAGIC publication (1ES 0033+595, 1ES 1011+496, B2 1215+30, and 1ES 1741+196) were, notably, later confirmed as VHE emitters, either during flaring activity, or by increasing the integration time.

\section{CONCLUSIONS}

The results from the analysis of the observations of non-detected blazars targeted by VERITAS from 2007 to 2012 have been presented. In addition, $\gamma$-ray sources from the 2FGL catalog which were within the field of view of these VERITAS observations were included in this study. For all the 114 sources included in this data-set we provided the VERITAS upper limit at VHE. Given that the redshift estimate of blazars is particularly important for VHE extragalactic astronomy, due to the $\gamma$-ray absorption on the EBL, we also presented the results from optical spectroscopy of 18 of these targets, determining the redshift for three of them, and providing a lower limit for the redshift of one of the sources.

We have presented the results from a stacked analysis of the data-set, showing that there is some evidence of signal with a significance level of $4 \sigma$.

In the near future, the sensitivity of VHE astronomy will be significantly increased thanks to the Cherenkov Telescope Array (CTA), which will be capable of detecting sources with VHE fluxes of the order of $0.001 \mathrm{Crab}$ units, about a factor of ten better than current IACTs (Actis et al. 2011). Among the scientific goals of CTA, an important endeavor will be to increase the number of known VHE blazars in order to perform population studies. Among the VERITAS targets presented in this work, the sources with the highest significance could be considered as primary candidates for observations with CTA, which may be able to detect many of them on the basis of the extrapolation of their Fermi-LAT spectra to higher energies. The non-detection of a number of later detected VHE blazars emphasizes the variable nature of these sources, highlighting the importance of monitoring observations in order to increase the likelihood of catching the sources at detectable VHE states.

This research is supported by grants from the U.S. Department of Energy Office of Science, the U.S. National Science Foundation and the Smithsonian Institution, and by NSERC in Canada. We acknowledge the excellent work of the technical support staff at the Fred Lawrence Whipple Observatory and at the collaborating institutions in the construction and operation of the instrument. The VERITAS Collaboration is grateful to Trevor Weekes for his seminal contributions and leadership in the field of VHE gamma-ray astrophysics, which made this study possible. MF acknowledges support by the Science and Technology Facilities Council [grant number ST/L00075X/1].

\section{REFERENCES}

Abdo, A. A., Ackermann, M., Ajello, M., et al. 2009a, ApJS, 183, 46 -. 2009b, ApJ, 707, L142

-. 2010a, ApJS, 188, 405

Abdo, A. A., Ackermann, M., Agudo, I., et al. 2010b, ApJ, 716, 30

Abeysekara, A. U. 2015, to appear in Proceedings of the International Cosmic Ray Conference, ArXiv e-prints 1508.06334

Abeysekara, A. U., Archambault, S., Archer, A., et al. 2015, ApJ, 815, L22 Abramowski, A., Acero, F., Aharonian, F., et al. 2013a, A\&A, 554, A107 -. 2013b, A\&A, 550, A4

Abramowski, A., Aharonian, F., Ait Benkhali, F., et al. 2014, A\&A, 564, A9 Acciari, V. A., Aliu, E., Arlen, T., et al. 2010, ApJ, 708, L100

Ackermann, M., Ajello, M., Allafort, A., et al. 2012a, ApJ, 753, 83

Ackermann, M., Ajello, M., Ballet, J., et al. 2012b, ApJ, 751, 159

Actis, M., Agnetta, G., Aharonian, F., et al. 2011, Experimental Astronomy, 32,193

Afanas'Ev, V. L., Dodonov, S. N., Moiseev, A. V., et al. 2005, Astronomy

Reports, 49, 374

Aharonian, F., Akhperjanian, A., Beilicke, M., et al. 2004, A\&A, 421, 529

Aharonian, F., Akhperjanian, A. G., Bazer-Bachi, A. R., et al. 2005, A\&A, 441,465

Aharonian, F., Akhperjanian, A. G., Barres de Almeida, U., et al. 2008a, A\&A, 481, L103

- 2008b, A\&A, 478, 387

Ahnen, M. L., Ansoldi, S., Antonelli, L. A., et al. 2015, ApJ, 815, L23

Ajello, M., Romani, R. W., Gasparrini, D., et al. 2014, ApJ, 780, 73

Albert, J., Aliu, E., Anderhub, H., et al. 2008, Science, 320, 1752

Aleksić, J., Antonelli, L. A., Antoranz, P., et al. 2011a, ApJ, 729, 115

-. 2011b, ApJ, 730, L8

Aleksić, J., Alvarez, E. A., Antonelli, L. A., et al. 2012, A\&A, 539, A118

Aleksić, J., Ansoldi, S., Antonelli, L. A., et al. 2014a, A\&A, 572, A121 

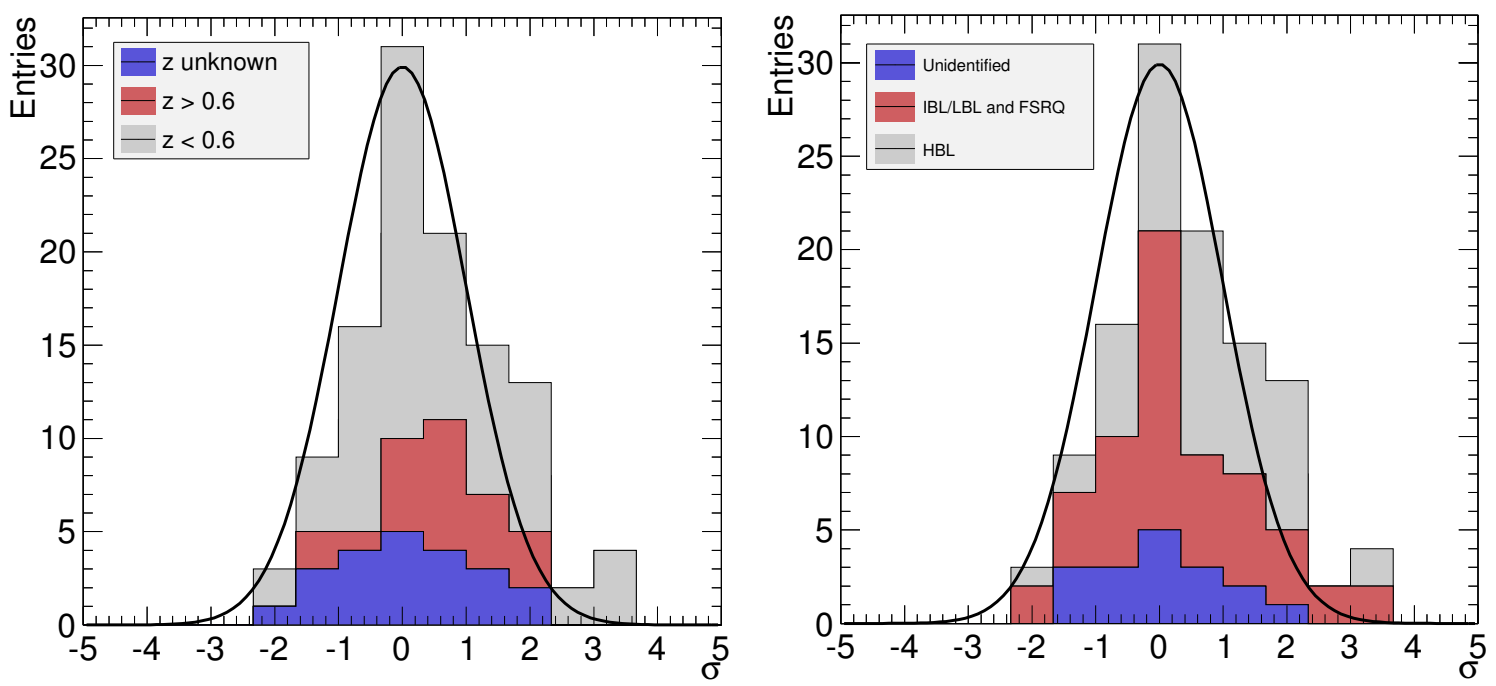

Figure 1. Left: stacked significance distribution of the sources included in our sample, classified according to their redshift. Sources with unknown $\mathrm{z}$ are in blue, sources with $z>0.6$ are in red and sources with $z<0.6$ are in grey. The Gaussian function represents the expectation from a randomly distributed sample, with mean equal to zero, and variance equal to 1. Right: same as left panel, but for sources classified according to the AGN type. Unidentified sources are in blue, IBL/LBL/FSRQ in red and HBL in grey.

-. 2014b, A\&A, 569, A46

-. 2014c, A\&A, 567, A41

-. 2015a, MNRAS, 446, 217

-. 2015b, Journal of High Energy Astrophysics, 5, 30

Aliu, E., Archambault, S., Arlen, T., et al. 2012, ApJ, 759, 102

Angel, J. R. P., \& Stockman, H. S. 1980, ARA\&A, 18, 321

Archambault, S., Arlen, T., Aune, T., et al. 2013, ApJ, 776, 69

Archambault, S., Aune, T., Behera, B., et al. 2014, ApJ, 785, L16

Archambault, S., Archer, A., Beilicke, M., et al. 2015, ApJ, 808, 110

Atwood, W. B., Abdo, A. A., Ackermann, M., et al. 2009, ApJ, 697, 1071

Bade, N., Fink, H. H., \& Engels, D. 1994, A\&A, 286, 381

Bauer, F. E., Condon, J. J., Thuan, T. X., \& Broderick, J. J. 2000, ApJS, 129 547

Benbow, W. 2015, to appear in Proceedings of the International Cosmic Ray Conference, ArXiv e-prints 1508.07251

Bird, R. 2015, to appear in Proceedings of the International Cosmic Ray Conference, ArXiv e-prints 1508.07195

Böhringer, H., Voges, W., Huchra, J. P., et al. 2000, ApJS, 129, 435

Brinkmann, W., Siebert, J., Feigelson, E. D., et al. 1997, A\&A, 323, 739

Burbidge, E. M., \& Kinman, T. D. 1966, ApJ, 145, 654

Burbidge, G. R., Crowne, A. H., \& Smith, H. E. 1977, ApJS, 33, 113

Şentürk, G. D., Errando, M., Böttcher, M., \& Mukherjee, R. 2013, ApJ, 764 119

Cao, L., Wei, J.-Y., \& Hu, J.-Y. 1999, A\&AS, 135, 243

Carswell, R. F., Strittmatter, P. A., Williams, R. E., Kinman, T. D., \& Serkowski, K. 1974, ApJ, 190, L101

Chandra, S., Baliyan, K. S., Ganesh, S., \& Joshi, U. C. 2012, ApJ, 746, 92

Cohen, R. D., Smith, H. E., Junkkarinen, V. T., \& Burbidge, E. M. 1987, ApJ, 318, 577

Cornwell, T. J., Saikia, D. J., Shastri, P., et al. 1986, Journal of Astrophysics and Astronomy, 7, 119

Cortina, J. 2013, The Astronomer's Telegram, 5080, 1

Costamante, L. 2007, Ap\&SS, 309, 487

Costamante, L., \& Ghisellini, G. 2002, A\&A, 384, 56

Doert, M., \& Errando, M. 2014, ApJ, 782, 41

Drinkwater, M. J., Webster, R. L., Francis, P. J., et al. 1997, MNRAS, 284 85

Eracleous, M., \& Halpern, J. P. 2004, ApJS, 150, 181

Errando, M. 2011, International Cosmic Ray Conference, 8, 133

Falcone, A. D., Bond, I. H., Boyle, P. J., et al. 2004, ApJ, 613, 710

Falomo, R. 1991, AJ, 102, 1991

Falomo, R., \& Kotilainen, J. K. 1999, A\&A, 352, 85

Fanaroff, B. L., \& Riley, J. M. 1974, MNRAS, 167, 31P

Fischer, J.-U., Hasinger, G., Schwope, A. D., et al. 1998, Astronomische Nachrichten, 319, 347

Fomin, V. P., Stepanian, A. A., Lamb, R. C., et al. 1994, Astroparticle Physics, 2, 137

Franceschini, A., Rodighiero, G., \& Vaccari, M. 2008, A\&A, 487, 837 Fumagalli, M., Dessauges-Zavadsky, M., Furniss, A., et al. 2012, MNRAS, 424, 2276

Furniss, A., Williams, D. A., Danforth, C., et al. 2013, ApJ, 768, L31

Ghisellini, G., Tagliaferri, G., Foschini, L., et al. 2011, MNRAS, 411, 901

Giommi, P., Piranomonte, S., Perri, M., \& Padovani, P. 2005, A\&A, 434, 385
Giommi, P., Polenta, G., Lähteenmäki, A., et al. 2012, A\&A, 541, A160

Glikman, E., Helfand, D. J., White, R. L., et al. 2007, ApJ, 667, 673

Halpern, J. P., Impey, C. D., Bothun, G. D., et al. 1986, ApJ, 302, 711

Healey, S. E., Romani, R. W., Cotter, G., et al. 2008, ApJS, 175, 97

Henstock, D. R., Browne, I. W. A., Wilkinson, P. N., \& McMahon, R. G.

1997, MNRAS, 290, 380

Hewitt, A., \& Burbidge, G. 1993, ApJS, 87, 451

Hillas, A. M. 1985, International Cosmic Ray Conference, 3, 445

Hillas, A. M., Akerlof, C. W., Biller, S. D., et al. 1998, ApJ, 503, 744

Holder, J. 2011, International Cosmic Ray Conference, 12, 137

- 2014a, The Astronomer's Telegram, 5981,

-. 2014b, The Astronomer's Telegram, 6849,

Holder, J., Atkins, R. W., Badran, H. M., et al. 2006, Astroparticle Physics, 25,391

Horan, D., Badran, H. M., Bond, I. H., et al. 2004, ApJ, 603, 51

Impey, C. D., \& Neugebauer, G. 1988, AJ, 95, 307

Jannuzi, B. T., Smith, P. S., \& Elston, R. 1993, ApJS, 85, 265

Kieda, D. B. 2013, Proceedings of the 33rd International Cosmic Ray

Conference

Komossa, S., Voges, W., Xu, D., et al. 2006, AJ, 132, 531

Konigl, A. 1981, ApJ, 243, 700

Kraus, J. D., \& Gearhart, M. R. 1975, AJ, 80, 1

Landoni, M., Falomo, R., Treves, A., \& Sbarufatti, B. 2014, A\&A, 570, A126

Laurent-Muehleisen, S. A., Kollgaard, R. I., Ciardullo, R., et al. 1998, ApJS, 118,127

Laurent-Muehleisen, S. A., Kollgaard, R. I., Feigelson, E. D., Brinkmann,

W., \& Siebert, J. 1999, ApJ, 525, 127

Lavaux, G., \& Hudson, M. J. 2011, MNRAS, 416, 2840

Lawrence, C. R., Pearson, T. J., Readhead, A. C. S., \& Unwin, S. C. 1986, AJ, 91, 494

Li, H. Z., Xie, G. Z., Yi, T. F., Chen, L. E., \& Dai, H. 2010, ApJ, 709, 1407

Li, T.-P., \& Ma, Y.-Q. 1983, ApJ, 272, 317

Lister, M. L., Aller, M., Aller, H., et al. 2011, ApJ, 742, 27

Marcha, M. J. M., Browne, I. W. A., Impey, C. D., \& Smith, P. S. 1996, MNRAS, 281, 425

Marziani, P., Sulentic, J. W., Dultzin-Hacyan, D., Calvani, M., \& Moles, M. 1996, ApJS, 104, 37

Maselli, A., Massaro, E., Nesci, R., et al. 2010, A\&A, 512, A74

Massaro, E., Giommi, P., Leto, C., et al. 2009, A\&A, 495, 691

Massaro, E., Giommi, P., Perri, M., et al. 2003, A\&A, 399, 33

Massaro, F., D’Abrusco, R., Tosti, G., et al. 2012, ApJ, 750, 138

Meisner, A. M., \& Romani, R. W. 2010, ApJ, 712, 14

Mirabal, N., Frías-Martinez, V., Hassan, T., \& Frías-Martinez, E. 2012 MNRAS, 424, L64

Mirzoyan, R. 2014a, The Astronomer's Telegram, 6062, 1

- 2014b, The Astronomer's Telegram, 6349, 1

- 2014c, The Astronomer's Telegram, 5768, 1

Mücke, A., \& Protheroe, R. J. 2001, Astroparticle Physics, 15, 121

Mukherjee, R. 2001, in American Institute of Physics Conference Series,

Vol. 558, American Institute of Physics Conference Series, ed. F. A.

Aharonian \& H. J. Völk, 324-337

Muriel, H., Donzelli, C., Rovero, A. C., \& Pichel, A. 2015, A\&A, 574, A101 
Nieppola, E., Tornikoski, M., \& Valtaoja, E. 2006, A\&A, 445, 441

Nilsson, K., Pursimo, T., Heidt, J., et al. 2003, A\&A, 400, 95

Nolan, P. L., Abdo, A. A., Ackermann, M., et al. 2012, ApJS, 199, 31

Osterbrock, D. E., \& Dahari, O. 1983, ApJ, 273, 478

Padovani, P. 1992, A\&A, 256, 399

Padovani, P., Costamante, L., Ghisellini, G., Giommi, P., \& Perlman, E. 2002, ApJ, 581, 895

Padovani, P., \& Giommi, P. 1995a, MNRAS, 277, 1477

- 1995 b, ApJ, 444, 567

Perlman, E. S. 2000, in American Institute of Physics Conference Series, Vol. 515, American Institute of Physics Conference Series, ed. B. L. Dingus, M. H. Salamon, \& D. B. Kieda, 53-65

Perlman, E. S., Stocke, J. T., Schachter, J. F., et al. 1996, ApJS, 104, 251 Piranomonte, S., Perri, M., Giommi, P., Landt, H., \& Padovani, P. 2007, A\&A, 470, 787

Piron, F. 2000, PhD thesis, Université de Paris-Sud (Paris XI)

Plotkin, R. M., Anderson, S. F., Brandt, W. N., et al. 2010, AJ, 139, 390

Rani, B., Gupta, A. C., Bachev, R., et al. 2011, MNRAS, 417, 1881

Rau, A., Schady, P., Greiner, J., et al. 2012, A\&A, 538, A26

Rolke, W. A., López, A. M., \& Conrad, J. 2005, Nuclear Instruments and Methods in Physics Research A, 551, 493

Salamon, M. H., \& Stecker, F. W. 1998, ApJ, 493, 547

Sandrinelli, A., Treves, A., Falomo, R., et al. 2013, AJ, 146, 163

Sbarufatti, B., Treves, A., Decarli, R., et al. 2009, The Astronomer's Telegram, 2123, 1

Sbarufatti, B., Treves, A., Falomo, R., et al. 2005, AJ, 129, 559
-. 2006, AJ, 132, 1

Seta, H., Isobe, N., Tashiro, M. S., et al. 2009, PASJ, 61, 1011

Shaw, M. S., Romani, R. W., Healey, S. E., et al. 2009, ApJ, 704, 477

Shaw, M. S., Romani, R. W., Cotter, G., et al. 2012, ApJ, 748, 49

-. 2013, ApJ, 764, 135

Sikora, M. Begelman, M. C., \& Rees, M. J. 1994, ApJ, 421, 153

Smith, H. E., Smith, E. O., \& Spinrad, H. 1976, PASP, 88, 621

Sol, H., Zech, A., Boisson, C., et al. 2013, Astroparticle Physics, 43, 215

Sowards-Emmerd, D., Romani, R. W., Michelson, P. F., Healey, S. E., \& Nolan, P. L. 2005, ApJ, 626, 95

Stecker, F. W., de Jager, O. C., \& Salamon, M. H. 1996, ApJ, 473, L75

Stickel, M., Fried, J. W., \& Kuehr, H. 1988, A\&A, 191, L16

-. 1989, A\&AS, 80, 103

Stickel, M., Padovani, P., Urry, C. M., Fried, J. W., \& Kuehr, H. 1991, ApJ, 374,431

Stocke, J. T., Morris, S. L., Gioia, I. M., et al. 1991, ApJS, 76, 813

Taylor, A. M., Vovk, I., \& Neronov, A. 2011, A\&A, 529, A144

Thompson, D. J., Djorgovski, S., \& de Carvalho, R. 1990, PASP, 102, 1235

Turriziani, S., Cavazzuti, E., \& Giommi, P. 2007, A\&A, 472, 699

Urry, C. M., \& Padovani, P. 1995, PASP, 107, 803

White, G. L., Jauncey, D. L., Wright, A. E., et al. 1988, ApJ, 327, 561

White, R. L., Becker, R. H., Gregg, M. D., et al. 2000, ApJS, 126, 133

Williams, D. A. 2005, in American Institute of Physics Conference Series,

Vol. 745, High Energy Gamma-Ray Astronomy, ed. F. A. Aharonian, H. J.

Völk, \& D. Horns, 499-504 


\section{APPENDIX}

\section{A. NEW REDSHIFT ESTIMATES}

In this appendix, we present optical spectra of 18 blazars taken at Lick Observatory in an attempt to spectroscopically measure their redshift. The sources selected for spectroscopy were selected independently from the sources selected in the main text, so the overlap is not complete. The spectra we show were taken between August, 2010 and October, 2014. During the observations at Lick we often observed the same source more than once. Duplicate observations are noted in Table A1, but we only show one spectrum per source in the figures. Four of these spectra (shown in Figure A1) have host galaxy features which allow an accurate redshift determination, and are discussed in the main text.

Table A1

Blazars observed at Lick Observatory using the Shane 3m Kast Spectrograph

\begin{tabular}{|c|c|c|c|c|c|c|c|}
\hline Target Name ${ }^{a}$ & $\begin{array}{c}\text { Obs. Date } \\
\text { [UT] }\end{array}$ & $\begin{array}{c}\text { Obs. Date } \\
\text { [MJD - 50000] }\end{array}$ & $\begin{array}{c}\text { Exposure } \\
{[\mathrm{s}]}\end{array}$ & Signal to noise ${ }^{b}$ & Standard Star & $\mathrm{z}$ & Fig. ${ }^{c}$ \\
\hline RBS 0082 & August 13,2010 & 5421 & 3600 & 50,77 & $\mathrm{BD}+284211$ & & $\overline{\mathrm{A} 2}$ \\
\hline 1ES $0033+595$ & August 22, 2012 & 6161 & 3600 & 20,88 & $\mathrm{BD}+284211$ & & \\
\hline 1ES $0033+595$ & December 4, 2013 & 6630 & 3600 & $2.8,34$ & G191B2B & & $\mathrm{A} 2$ \\
\hline 1RXS J0045.3+2127 & August 22, 2012 & 6161 & 1800 & 56,106 & $\mathrm{BD}+284211$ & & \\
\hline 1RXS J0045.3+2127 & October 28,2014 & 6958 & 1800 & 57,121 & Feige 110 & & $\mathrm{~A} 2$ \\
\hline RGB J0250+172 & August 15, 2010 & 5423 & 5400 & 21,44 & $\mathrm{BD}+284211$ & 0.243 & A1 \\
\hline $1 \mathrm{ES} 0446+449$ & February 14,2013 & 6337 & 3600 & $\mathrm{n} / \mathrm{a}, 65$ & $\mathrm{HZ} 2$ & & A3 \\
\hline RGB J0505+612 & February 14, 2013 & 6337 & 900 & $0.4,4.3$ & HZ2 & & A3 \\
\hline 2FGL J0540.4+5822 & October 28,2014 & 6958 & 3600 & 14,46 & G19B2B & & A3 \\
\hline B2 $0912+29$ & April 7, 2013 & 6389 & 3600 & 57,179 & Feige 34 & & \\
\hline B2 $0912+29$ & January 4, 2013 & 6299 & 3600 & 81,144 & Feige 34 & & A4 \\
\hline B2 $0912+29$ & December 4, 2013 & 6630 & 3600 & 48,102 & G191B2B & & \\
\hline RBS 0929 & April 7, 2013 & 6389 & 3600 & 13,33 & Feige 34 & & A4 \\
\hline RGB J1037+571 & February 14, 2013 & 6337 & 3600 & 54,128 & Feige 34 & & A4 \\
\hline 1ES 1118+424 & February 14, 2013 & 6337 & 3600 & $1.5,22$ & Feige 34 & 0.230 & AI \\
\hline PG $1246+586$ & April 7, 2013 & 6389 & 3600 & 80,229 & GD 153 & & \\
\hline PG $1246+586$ & May 29, 2014 & 6806 & 3600 & 86,175 & HZ44 & & \\
\hline PG $1246+586$ & May 30,2014 & 6807 & 3600 & 80,203 & HZ44 & & A5 \\
\hline RBS 1366 & April 7, 2013 & 6389 & 900 & 7,34 & $\mathrm{BD}+332642$ & & \\
\hline RBS 1366 & May 30, 2014 & 6807 & 3600 & 26,72 & BD+33 2642 & 0.237 & A1 \\
\hline 1RXS J144053.2+061013 & January 4, 2013 & 6299 & 3800 & 13,53 & BD+33 2642 & & A5 \\
\hline RGB J1725+118 & June 12, 2013 & 6455 & 3600 & 48,129 & $\mathrm{BD}+332642$ & & \\
\hline RGB J1725+118 & May 29, 2014 & 6806 & 3600 & 81,189 & $\mathrm{BD}+332642$ & & \\
\hline RGB J1725+118 & May 30,2014 & 6807 & 3600 & 111,205 & $\mathrm{BD}+332642$ & & A5 \\
\hline RGB J1903+556 & June 13, 2013 & 6456 & 1800 & 18,46 & $\mathrm{BD}+284211$ & & Aర \\
\hline BZB J2243+2021 & August 13, 2010 & 5421 & 3600 & 92,167 & $\mathrm{BD}+284211$ & & $\overline{\mathrm{AO}}$ \\
\hline 1ES $2321+419$ & August 22, 2012 & 6161 & 3000 & 20,33 & $\mathrm{BD}+284211$ & & \\
\hline 1ES $2321+419$ & October 28,2014 & 6958 & 3600 & 52,121 & Feige 110 & $>0.267$ & A1 \\
\hline
\end{tabular}

Note. $-{ }^{a}$ See Table 1 in main text for coordinates.

${ }^{b}$ This is the average signal to noise per pixel. The first number is for the blue side CCD, between 3500 and $5400 \mathrm{~A}$. The second number is for the red side CCD, between 5700 and 6800 Angstroms

${ }^{c}$ Several targets have spectra from multiple nights. Only one spectrum per target is shown; this column indicates the corresponding figure, if applicable. 

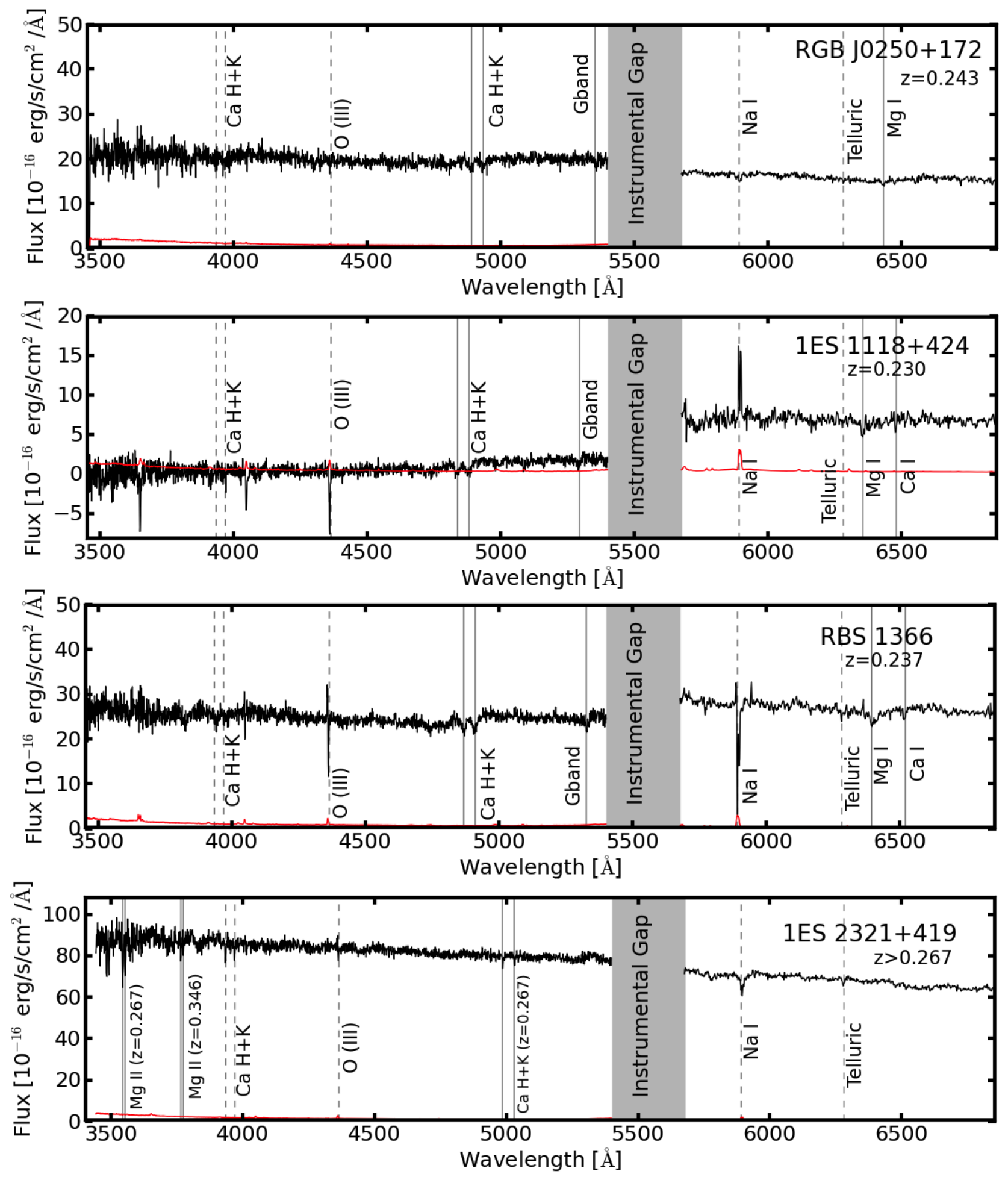

Figure A1. Spectra shown from top to bottom: RGB J0250+172 (August 15, 2010), 1ES 1118+424 (February 14, 2013 ), RBS 1366 (May 30, 2014), 1ES $2321+419$ (October 28, 2014). Dashed lines indicate telluric and Galactic features. Red lines indicate the error array for each observation; some are not visible due to high $\mathrm{S} / \mathrm{N}$. Solid gray lines indicate features at non-zero redshift. 

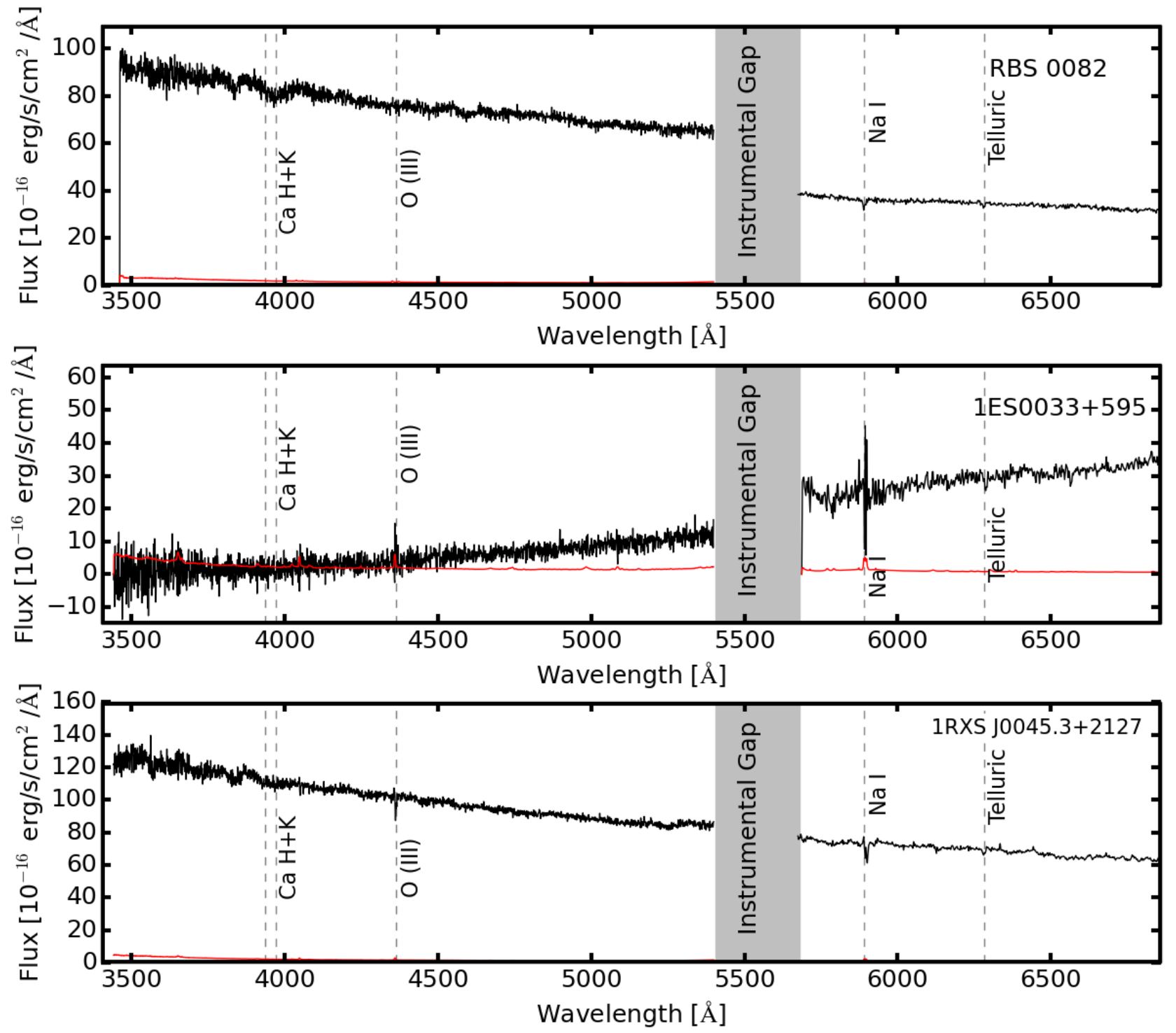

Figure A2. Spectra shown from top to bottom: RBS 0082 (August 13, 2010), 1ES 0033+595 (December 4, 2013), 1RXS J0045.3+2127 (October 28, 2014 ). Dashed lines indicate telluric and Galactic features. Red lines indicate the error array for each observation; some are not visible due to high S/N. 

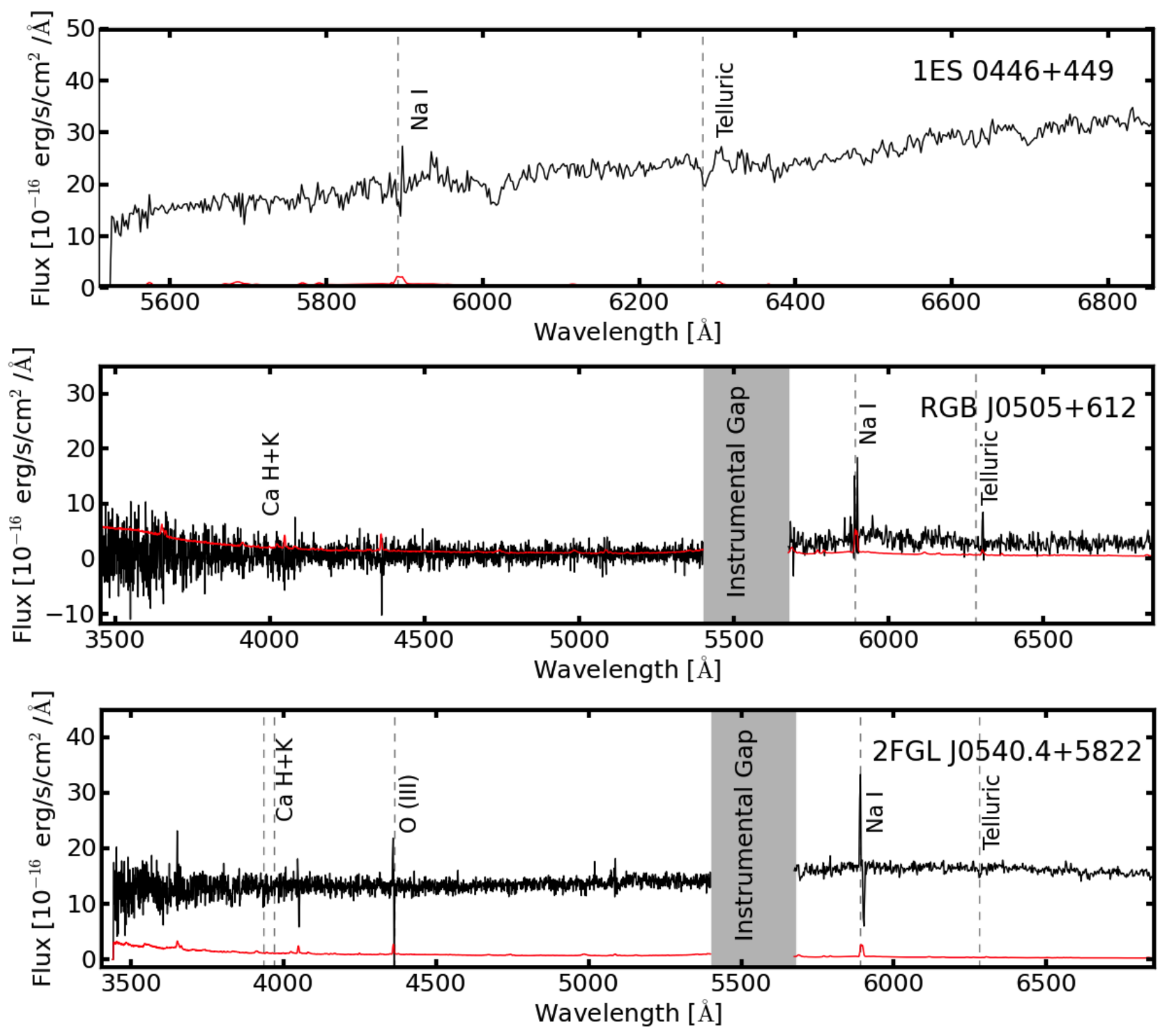

Figure A3. Spectra shown from top to bottom: 1ES 0446+449 (February 14, 2013), RGB J0505+612 (February 14, 2013), 2FGL J0540.4+5822 (October 28, 2014). The spectrum of 1ES 0446+449 (February 14, 2013) only includes the red arm data (5500-8000 Angstroms) because there were complications in reducing the blue arm data. Dashed lines indicate telluric and Galactic features. Red lines indicate the error array for each observation; some are not visible due to high $\mathrm{S} / \mathrm{N}$. 

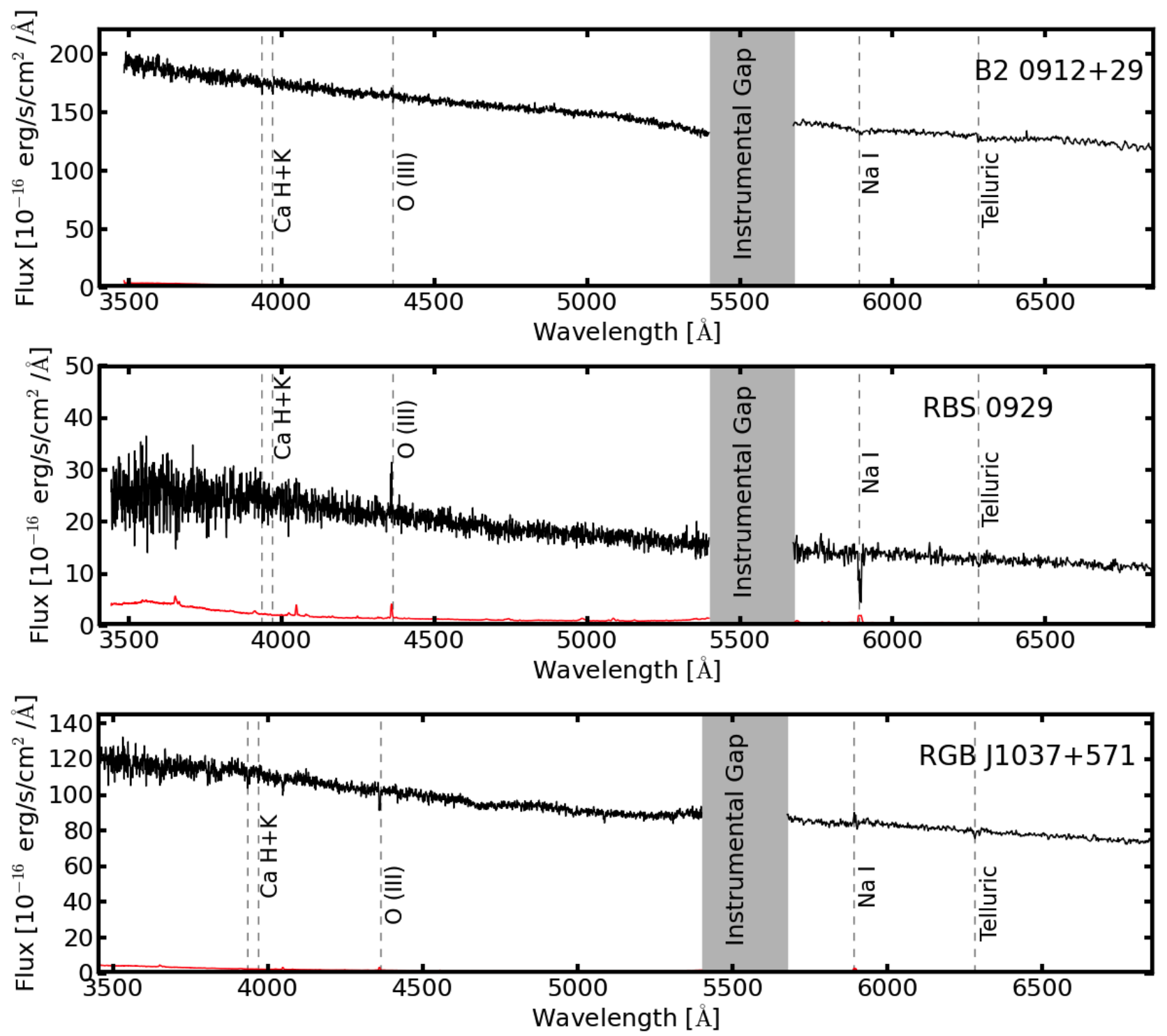

Figure A4. Spectra shown from top to bottom: B2 0912+29 (January 4, 2013), RBS 0929 (April 7, 2013), RGB J1037+571 (February 14, 2013 ). Dashed lines indicate telluric and Galactic features. Red lines indicate the error array for each observation; some are not visible due to high S/N. 

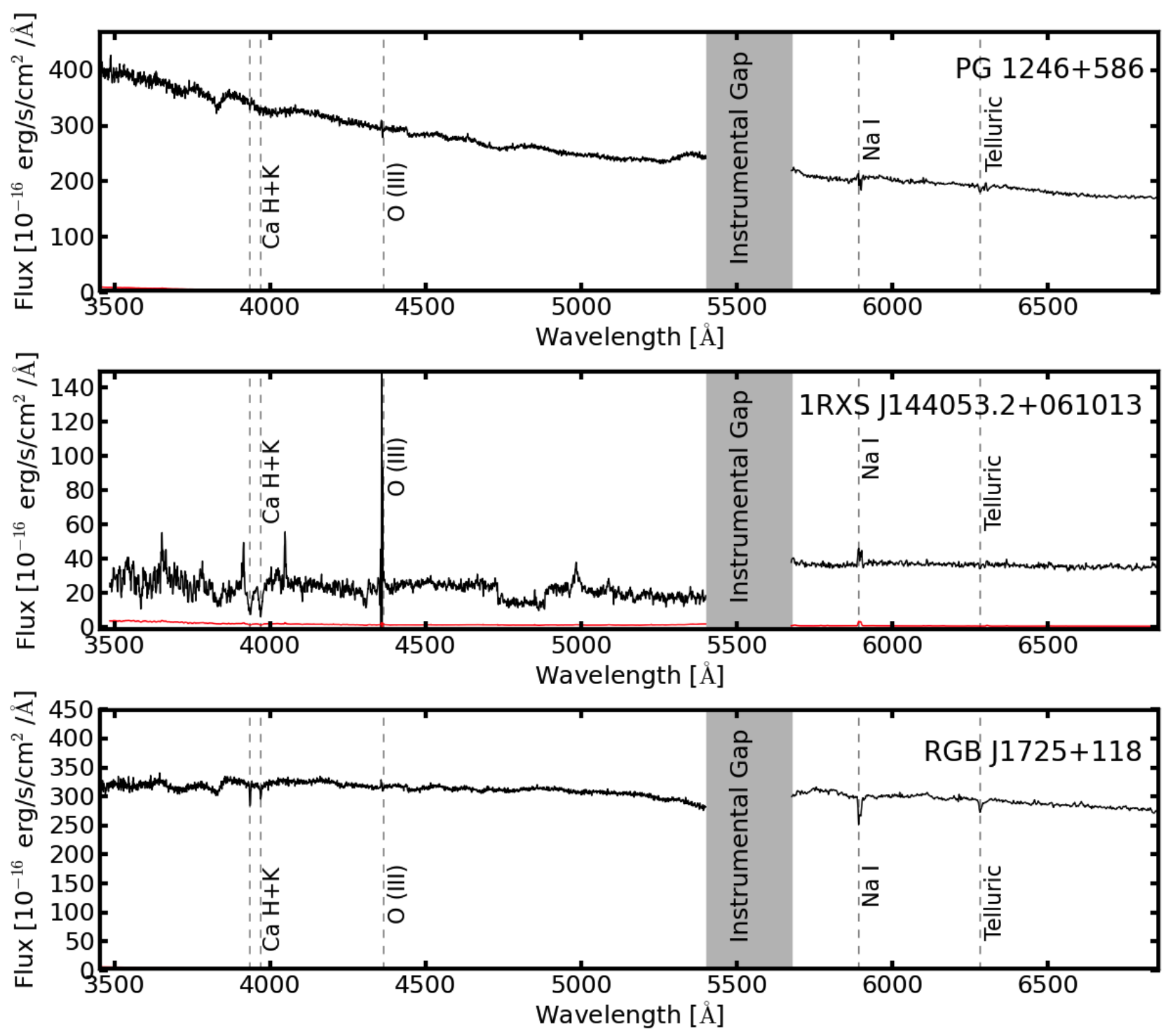

Figure A5. Spectra shown from top to bottom: PG 1246+586 (May 30, 2014), 1RXS J144053.2+061013 (January 4, 2013), RGB J1725+118 (May 30, 2014). Dashed lines indicate telluric and Galactic features. Red lines indicate the error array for each observation; some are not visible due to high S/N. 

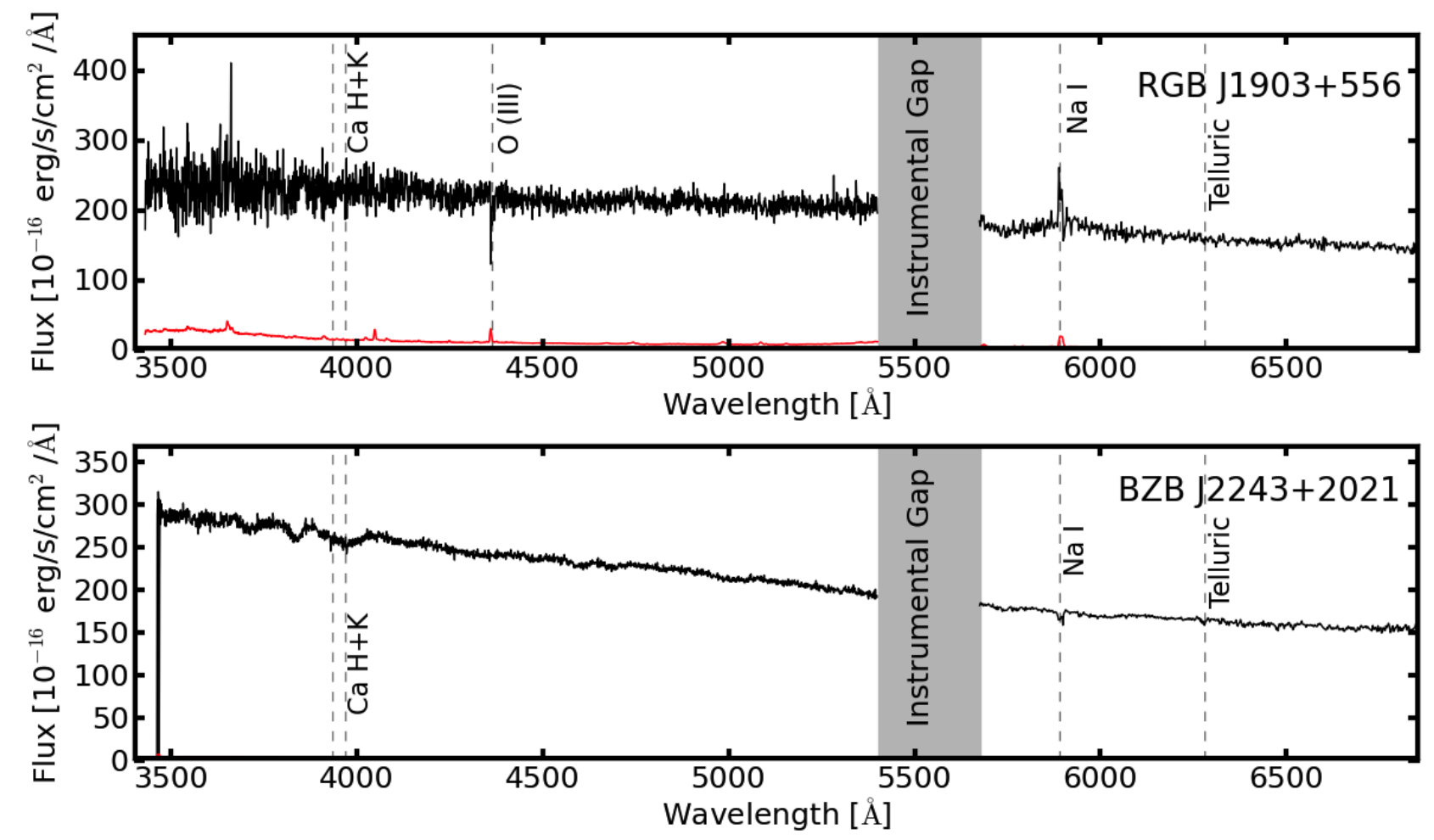

Figure A6. Spectra shown from top to bottom: RGB J1903+556 (June 13, 2013), BZB J2243+2021 (August 13, 2010). Dashed lines indicate telluric and Galactic features. Red lines indicate the error array for each observation; some are not visible due to high S/N. 